Georgian Mathematical Journal

1(1994), No. 6, 641-673

\title{
CRITERIA OF WEIGHTED INEQUALITIES IN ORLICZ CLASSES FOR MAXIMAL FUNCTIONS DEFINED ON HOMOGENEOUS TYPE SPACES
}

\author{
A. GOGATISHVILI AND V. KOKILASHVILI
}

\begin{abstract}
The necessary and sufficient conditions are derived in order that a strong type weighted inequality be fulfilled in Orlicz classes for scalar and vector-valued maximal functions defined on homogeneous type space. A weak type problem with weights is solved for vector-valued maximal functions.
\end{abstract}

\section{$\S 0$. INTRODUCTION}

The main goal of this paper is to obtain criteria for the validity of an inequality of the form

$$
\int_{X} \varphi(\mathbf{M} f(x)) w(x) d \mu \leq c \int_{X} \varphi(f(x)) w(x) d \mu
$$

for maximal functions defined on homogeneous type spaces.

The solution of a strong type one-weighted problem for classical maximal functions in reflexive Orlicz spaces was obtained for the first time by $\mathrm{R}$. Kerman and A. Torchinsky [5]. This investigation was further developed in [6], [7]). Quite a simple criterion established in this paper in the general case is the new one for Hardy-Littlewood-Wiener maximal functions as well. Our present investigation is a natural continuation of the non-weighted case [1], [2], [3], [4]. Conceptually it is close to [2], [8], [9], [15], [16].

For vector-valued Hardy-Littlewood-Wiener maximal functions in the non-weighted case the boundedness in $L^{p}, 1<p<\infty$, was established in [9]. A weighted analogue of this result was obtained in [10] (see also [11], [12], [13]). Finally, we should mention [14], [15], [16] containing the full descriptions of functions $\varphi$ and a set of weight functions ensuring the validity of a weak type weighted inequality for maximal functions.

1991 Mathematics Subject Classification. 42B25, 26D15, 46E30.

641

1072-947X/1994/1100-0641\$07.00/0 @ 1994 Plenum Publishing Corporation 
We shall now make some comments on how this paper is organized. The introduction contains some commonly known facts on homogeneous type spaces and weight functions defined in such spaces. Here the reader will also find the definition of quasi-convex functions and a brief discussion of some of their simple properties. The main results are formulated at the end of the introduction. In $\S 1$ we describe the class of quasi-convex functions, also functions which are quasi-convex to some degree less than 1 . A number of useful properties to be used in our further discussion are established for such functions. The further sections contain the proofs of the main results.

Let $(X, d, \mu)$ be a homogeneous type space (see, for example, [17], [19]). It is a metric space with a complete measure $\mu$ such that the class of compactly supported continuous functions is dense in the space $L^{1}(X, \mu)$. It is also assumed that there is a nonnegative real-valued function $d: X \times X \rightarrow \mathbb{R}^{1}$ satisfying the following conditions:

(i) $d(x, x)=0$ for all $x \in X$;

(ii) $d(x, y)>0$ for all $x \neq y$ in $X$;

(iii) there is a constant $a_{0}$ such that $d(x, y) \leq a_{0} d(y, x)$ for all $x, y$ in $X$;

(iv) there is a constant $a_{1}$ such that $d(x, y) \leq a_{1}(d(x, z)+d(z, y))$ for all $x, y, z$ in $X$;

(v) for each neighbourhood $V$ of $x$ in $X$ there is an $r>0$ such that the ball $B(x, r)=\{y \in X ; d(x, y)<r\}$ is contained in $V$;

(vi) the balls $B(x, r)$ are measurable for all $x$ and $r>0$;

(vii) there is a constant $b$ such that $\mu B(x, 2 r) \leq b \mu B(x, r)$ for all $x \in X$ and $r>0$.

An almost everywhere positive locally $\mu$-summable function $w: X \rightarrow \mathbb{R}^{1}$ will be called a weight function. For an arbitrary $\mu$-measurable set $E$ we shall assume

$$
w E=\int_{E} w(x) d \mu .
$$

By definition, the weight function $w \in \mathcal{A}_{p}(X)(1 \leq p<\infty)$ if

$$
\sup _{B}\left(\frac{1}{\mu B} \int_{B} w(x) d \mu\right)\left(\frac{1}{\mu B} \int_{B}(w(x))^{-1 /(p-1)} d \mu\right)^{p-1}<\infty \text { for } 1<p<\infty,
$$

where the supremum is taken over all balls $B \subset X$ and

$$
\frac{1}{\mu B} \int_{B} w(x) d \mu \leq \underset{y \in B}{\operatorname{essinf}} w(y) \text { for } p=1 .
$$

In the latter inequality $c$ does not depend on $B$. The above conditions are analogues of the well-known Muckenhoupt's conditions. 
Let us recall the basic properties of classes $\mathcal{A}_{p}$ (see [17], [20], [23]). If $w \in \mathcal{A}_{p}$ for some $p \in[1, \infty)$, then $w \in \mathcal{A}_{s}$ for all $s \in[p, \infty)$ and there is an $\varepsilon>0$ such that $w \in \mathcal{A}_{p-\varepsilon}$.

By definition, the weight function $w$ belongs to $\mathcal{A}_{\infty}(X)$ if to each $\varepsilon \in$ $(0,1)$ there corresponds $\delta \in(0,1)$ such that if $B \subset X$ is a ball and $E$ is any measurable set of $B$, then $\mu E<\delta \mu B$ implies $w E<\varepsilon w B$.

On account of the well-known properties of classes $\mathcal{A}_{p}$ we have

$$
\mathcal{A}_{\infty}(X)=\cup_{p \geq 1} \mathcal{A}_{p}(X)
$$

(see [17], [20], [21].)

In what follows we shall use the symbol $\Phi$ to denote the set of all functions $\varphi: \mathbb{R}^{1} \rightarrow \mathbb{R}^{1}$ which are nonnegative, even and increasing on $(0, \infty)$ such that $\varphi(0+)=0, \lim _{t \rightarrow \infty} \varphi(t)=\infty$. For our purpose we shall also need the following basic definition of quasi-convex functions:

A function $\omega$ is called a Young function on $[0, \infty)$ if $\omega(0)=0, \omega(\infty)=\infty$ and it is not identically zero or $\infty$ on $(0, \infty)$; it may have a jump up to $\infty$ at some point $t>0$ but in that case it should be left continuous at $t$ (see [18]).

A function $\varphi$ is called quasi-convex if there exist a Young function $\omega$ and a constant $c>1$ such that $\omega(t) \leq \varphi(t) \leq \omega(c t), t \geq 0$. Clearly, $\varphi(0)=0$ and for $s \leq t$ we have $\varphi(s) \leq \varphi(c t)$.

To each quasi-convex function $\varphi$ we can put into correspondence its complementary function $\widetilde{\varphi}$ defined by

$$
\widetilde{\varphi}(t)=\sup _{s \geq 0}(s t-\varphi(s)) .
$$

The subadditivity of the supremum readily implies that $\widetilde{\varphi}$ is always a Young function and $\widetilde{\varphi} \leq \varphi$. This equality holds if $\varphi$ itself is a Young function. If $\varphi_{1} \leq \varphi_{2}$, then $\widetilde{\varphi}_{2} \leq \widetilde{\varphi}_{1}$, and if $\varphi_{1}(t)=a \psi(b t)$ then

$$
\widetilde{\varphi}_{1}(t)=a \widetilde{\varphi}\left(\frac{t}{a b}\right) .
$$

Hence and from (0.2) we have

$$
\widetilde{\omega}\left(\frac{t}{c}\right) \leq \widetilde{\varphi}(t) \leq \widetilde{\omega}(t)
$$

Now from the definition of $\widetilde{\varphi}$ we obtain the Young inequality

$$
s t \leq \varphi(s)+\widetilde{\varphi}(t), \quad s, t \geq 0 .
$$

By definition, the function $\psi$ satisfies the global condition $\Delta_{2}\left(\psi \in \Delta_{2}\right)$ if there is $c>0$ such that $\psi(2 t) \leq c \psi(t), t>0$. 
If $\psi \in \Delta_{2}$, then there are $p>1$ and $c>1$ such that

$$
\frac{\psi\left(t_{2}\right)}{t_{2}^{p}} \leq \frac{c \psi\left(t_{1}\right)}{t_{1}^{p}} \text { for } 0<t_{1}<t_{2}
$$

(see [3], Lemma 1.3.2).

Given locally integrable real functions $f$ on $X$, we define the maximal function $\mathbf{M} f(x)$ by

$$
\mathbf{M} f(x)=\sup (\mu B)^{-1} \int_{B}|f(y)| d \mu, \quad x \in X,
$$

where the supremum is taken over all balls $B$ containing $x$.

As is well-known (see [20]), for the operator $\mathbf{M}: f \rightarrow \mathbf{M} f$ inequality (0.1) is fulfilled when $\varphi(u)=u^{p}(1<p<\infty)$ and $w \in \mathcal{A}_{p}(X)$. Now we are ready to formulate the main results of this paper.

Theorem I. Let $\varphi \in \Phi$. The following conditions are equivalent:

(i) there is a constant $c>0$ such that for any function $f: X \rightarrow R^{1}$ locally summable in the sense of $\mu$-measure we have the inequality

$$
\int_{x} \varphi(\mathbf{M} f(x)) w(x) d \mu \leq c \int_{X} \varphi(f(x)) w(x) d \mu,
$$

(ii) $\varphi^{\alpha}$ is quasi-convex for some $\alpha, 0<\alpha<1$, and $w \in \mathcal{A}_{p(\varphi)}$ where

$$
\frac{1}{p(\varphi)}=\inf \left\{\alpha: \varphi^{\alpha} \text { is quasi-convex }\right\} \text {. }
$$

Theorem II. Let $\varphi \in \Phi, 1<\theta<\infty$. In order that there exist a constant $c>0$ such that the inequality

$$
\begin{aligned}
& \int_{X} \varphi\left(\left(\sum_{i=1}^{\infty} \mathbf{M}^{\theta} f_{i}(x)\right)^{1 / \theta}\right) w(x) d \mu \leq \\
& \leq c \int_{X} \varphi\left(\left(\sum_{i=1}^{\infty}\left|f_{i}(x)\right|^{\theta}\right)^{1 / \theta}\right) w(x) d \mu
\end{aligned}
$$

be fulfilled for any vector-function $f=\left(f_{1}, f_{2}, \ldots\right)$ with locally summable components, it is necessary and sufficient that the following conditions be fulfilled: $\varphi \in \Delta_{2}, \varphi^{\alpha}$ is quasi-convex for some $\alpha, 0<\alpha<1$, and $w \in$ $\mathcal{A}_{p(\varphi)}$.

Theorem III. Let $\varphi \in \Phi$. Then the following conditions are equivalent: 
(i) there is a constant $c_{1}>0$ such that the inequality

$$
\int_{X} \varphi\left(\frac{\mathbf{M} f(x)}{w(x)}\right) w(x) d \mu \leq c_{1} \int_{X} \varphi\left(\frac{c_{1} f(x)}{w(x)}\right) w(x) d \mu
$$

holds for any $\mu$-measurable $f: X \rightarrow \mathbb{R}^{1}$;

(ii) $\varphi^{\alpha}$ is quasi-convex for some $\alpha \in(0,1)$ and $w \in \mathcal{A}_{p(\tilde{\varphi})}$;

(iii) $\varphi^{\alpha}$ is quasi-convex for some $\alpha \in(0,1)$ and there is a constant $c_{2}>0$ such that

$$
\widetilde{\varphi}\left(\frac{1}{\lambda \mu B} \int_{B} \varphi\left(\frac{\lambda}{w(x)}\right) w(x) d \mu\right) w B \leq c_{2} \int_{B} \varphi\left(\frac{\lambda}{w(x)}\right) w(x) d \mu
$$

for any $\lambda>0$ and ball $B$;

(iv) $\varphi^{\alpha}$ is quasi-convex for some $\alpha \in(0,1)$ and there exists a constant $c_{3}>0$ such that

$$
\int_{B} \varphi\left(\frac{\lambda w B}{w(x) \mu B}\right) w(x) d \mu \leq c_{3} \varphi(\lambda) w B
$$

for any $\lambda>0$ and ball $B$.

Theorem IV. Let $\varphi$ and $\gamma$ be nonnegative nondecreasing on $[0, \infty]$ functions. Further we suppose that $\psi$ is a quasi-convex function and $\psi \in \Delta_{2}$. If $0<\theta<1$, then the following conditions are equivalent:

(i) there exists a constant $c_{1}>0$ such that the inequality

$$
\begin{gathered}
\varphi(\lambda) w\left\{x \in X,\left(\sum_{i=1}^{\infty}\left(\mathbf{M} f_{i}(x)\right)^{\theta}\right)^{1 / \theta}>\lambda\right\} \leq \\
\leq c_{1} \int_{X} \psi\left(\frac{c_{1}}{\gamma(\lambda)}\left(\sum_{i=1}^{\infty}\left|f_{i}(x)\right|^{\theta}\right)^{1 / \theta}\right) w(x) d \mu
\end{gathered}
$$

is fulfilled for any $\lambda>0$ and vector-function $f=\left(f_{1}, \ldots, f_{n}, \ldots\right)$ with locally summable components;

(ii) there is a $\varepsilon>0$ such that

$$
\sup _{B} \sup _{s>0} \frac{1}{\varphi(s) w B} \int_{B} \widetilde{\psi}\left(\varepsilon \frac{\varphi(s) \gamma(s)}{s} \frac{w B}{\mu B w(x)}\right) w(x) d \mu<\infty .
$$

In this paper the letter $c$ may denote different positive constants which are independent of the meaningful variables in the present context. Throughout this paper we take $0 \cdot \infty$ to be zero. 


\section{$\S 1$. Some Properties of Quasi-convex Functions}

In this paragraph we describe the class of quasi-convex functions.

Lemma 1.1. Let $\varphi \in \Phi$. Then the following conditions are equivalent:

(i) $\varphi$ is quasi-convex;

(ii) there is a constant $c_{1}>0$ such that

$$
\frac{\varphi\left(t_{1}\right)}{t_{1}} \leq c_{1} \frac{\varphi\left(c_{1} t_{2}\right)}{t_{2}}
$$

is fulfilled for any $t_{1}$ and $t_{2}$ provided that $t_{1}<t_{2}$;

(iii) there is a constant $c_{2}>0$ such that

$$
\varphi(t) \leq \widetilde{\widetilde{\varphi}}\left(c_{2} t\right), \quad t>0
$$

(iv) there are positive $\varepsilon$ and $c_{3}$ such that

$$
\widetilde{\varphi}\left(\varepsilon \frac{\varphi(t)}{t}\right) \leq c_{3} \varphi(t), \quad t>0
$$

(v) there is a constant $c_{4}>0$ such that

$$
\varphi\left(\frac{1}{\mu B} \int_{B} f(y) d \mu\right) \leq \frac{c_{4}}{\mu B} \int_{B} \varphi\left(c_{4} f(y)\right) d \mu
$$

for any locally summable function $f$ and an arbitrary ball $B$.

Proof. For the equivalency of the conditions (i) and (ii) see [3], Lemma 1.1.1. We shall prove that the conditions (i) and (iii) are equivalent. Indeed, if the function $\varphi$ is quasi-convex, then for some convex function $\omega$ and constant $c_{2}$ we have $\varphi(t) \leq \omega\left(c_{2} t\right)=\widetilde{\widetilde{\omega}}\left(c_{2} t\right) \leq \widetilde{\widetilde{\varphi}}\left(c_{2} t\right)$. Conversely, let (iii) hold. The function $\widetilde{\widetilde{\varphi}}$ is convex and $\widetilde{\tilde{\varphi}} \leq \varphi$. Therefore by (iii) $\varphi(t) \leq \widetilde{\widetilde{\varphi}}\left(c_{2} t\right) \leq \varphi\left(c_{2} t\right)$, which means the quasi-convexity of the function $\varphi$.

Now we shall show that (i) $\Leftrightarrow$ (iv). The condition (i) implies that there is a convex function $\omega$ such that for some $c>0 \omega(t) \leq \varphi(t) \leq \omega(c t), t>0$. The function $\widetilde{\omega}$ is convex and $\widetilde{\varphi}(t) \leq \widetilde{\omega}(t)$. Therefore we have (see Lemmas 2.1 and 2.2 from [16])

$$
\widetilde{\varphi}\left(\varepsilon \frac{\varphi(t)}{t}\right) \leq \widetilde{\omega}\left(\varepsilon \frac{\varphi(t)}{t}\right) \leq \frac{\varphi(t)}{\omega(c t)} \widetilde{\omega}\left(\varepsilon c \frac{\omega(c t)}{c t}\right) \leq \varphi(t),
$$

provided that $c \varepsilon<1$. We have thereby proved the implication (i) $\Rightarrow$ (iv). Let us now assume that the condition (iv) holds. By the Young inequality 
we have for $s<t$

$$
\begin{gathered}
\frac{\varphi(s)}{s}=\frac{1}{2 c_{3} t} \varepsilon \frac{\varphi(s)}{s} \frac{2 c_{3}}{\varepsilon} t \leq \frac{1}{2 c_{3} t} \widetilde{\varphi}\left(\varepsilon \frac{\varphi(s)}{s}\right)+\frac{1}{2 c_{3} t} \varphi\left(\frac{2 c_{3}}{\varepsilon} t\right) \leq \\
\leq \frac{1}{2} \frac{\varphi(s)}{s}+\frac{1}{2 c_{3} t} \varphi\left(\frac{2 c_{3}}{\varepsilon} t\right) .
\end{gathered}
$$

Hence we obtain

$$
\frac{\varphi(s)}{s} \leq \frac{1}{c_{3} t} \varphi\left(\frac{2 c_{3}}{\varepsilon} t\right),
$$

which means the fulfilment of (ii) and, accordingly, of (i). The equivalency of the conditions (i) and (v) is proved as in [3], Lemma 1.1.1.

Corollary 1.1. For a quasi-convex function $\varphi$ we have the estimates

$$
\begin{aligned}
& \varepsilon \varphi(t) \leq \varphi(c \varepsilon t), \quad t>0, \quad \varepsilon>1, \\
& \varphi(\gamma t) \leq \gamma \varphi(c t), \quad t>0, \quad \gamma<1,
\end{aligned}
$$

where the constant $c$ does not depend on $t$.

Corollary 1.2. Let $\varphi \in \Phi$ and $\varphi$ be quasi-convex. Then there is a constant $\varepsilon>0$ such that for an arbitrary $t>0$ the following inequalities are fulfilled:

$$
\begin{aligned}
& \widetilde{\varphi}\left(\varepsilon \frac{\varphi(t)}{t}\right) \leq \varphi(t) \leq \widetilde{\varphi}\left(2 \frac{\varphi(t)}{t}\right), \\
& \varphi\left(\varepsilon \frac{\widetilde{\varphi}(t)}{t}\right) \leq \widetilde{\varphi}(t) \leq \varphi\left(2 \frac{\widetilde{\varphi}(t)}{t}\right) .
\end{aligned}
$$

Proof. The right-hand inequality of (1.5) is contained in Lemma 1.1. Further, the convexity of the function $\widetilde{\varphi}$ implies

$$
\widetilde{\varphi}\left(\frac{\tilde{\varphi}(t)}{t}\right) \leq \widetilde{\varphi}(t), \quad t>0
$$

while by Lemma 1.1 the quasi-convexity of the function $\varphi$ implies

$$
\varphi(t) \leq \widetilde{\widetilde{\varphi}}(c t), \quad t>0
$$

for some $c>0$. Therefore, choosing $\varepsilon>0$ such that $c \varepsilon<1$, we obtain

$$
\varphi\left(\varepsilon \frac{\widetilde{\varphi}(t)}{t}\right) \leq \widetilde{\varphi}\left(c \varepsilon \frac{\widetilde{\varphi}(t)}{t}\right) \leq \widetilde{\varphi}\left(\frac{\widetilde{\varphi}(t)}{t}\right) \leq \widetilde{\varphi}(t),
$$

thereby proving the left-hand inequality of (1.6).

Next, by virtue of the Young inequality

$$
\varphi(t) \leq \frac{1}{2} \widetilde{\varphi}\left(2 \frac{\varphi(t)}{t}\right)+\frac{1}{2} \varphi(t) .
$$


Hence

$$
\varphi(t) \leq \widetilde{\varphi}\left(2 \frac{\varphi(t)}{t}\right)
$$

Analogously, we obtain

$$
\widetilde{\varphi}(t) \leq \varphi\left(2 \frac{\widetilde{\varphi}(t)}{t}\right),
$$

thereby also proving the right-hand sides of inequalities (1.5) and (1.6).

Lemma 1.2. Let $\varphi \in \Phi$. Then the following conditions are equaivalent:

(i) the function $\varphi^{\alpha}$ is quasi-convex for some $\alpha, 0<\alpha<1$;

(ii) the function $\varphi$ is quasi-convex and $\widetilde{\varphi} \in \Delta_{2}$;

(iii) there is a $a>1$ such that

$$
\varphi(a t) \geq 2 a \varphi(t), \quad t>0
$$

(iv) there is a constant $c>0$ such that for any $t$ we have

$$
\int_{0}^{t} \frac{\varphi(s)}{s^{2}} d s \leq c \frac{\varphi(c t)}{t} .
$$

Proof. The equivalency of the conditions (i), (iii) and (iv) is proved in [3] (Theorem 1.2.1). It remains for us to assume that each of these conditions is equivalent to the condition (ii). We shall show that (ii) $\Leftrightarrow($ iii). Assume that (iii) holds. Then

$$
\begin{gathered}
\widetilde{\varphi}(2 t)=\sup _{s \geq 0}(2 t s-\varphi(s))=\sup _{s \geq 0}(2 a t s-\varphi(a s)) \leq \\
\leq \sup _{s \geq 0}(2 a t s-2 a \varphi(s))=2 a \widetilde{\varphi}(t) .
\end{gathered}
$$

Let now $\widetilde{\varphi}(2 t) \leq c_{1} \widetilde{\varphi}(t)$ for some constant $c_{1}$ and an arbitrary $t>0$. Since $\varphi$ is quasi-convex, then by Lemma 1.1 $\widetilde{\widetilde{\varphi}}(c t) \geq \varphi(t)$ for some $c>0$ and any $t>0$.

For the constant $a_{1}$ with the condition $2 a_{1}>c_{1}$ we have

$$
\begin{aligned}
\widetilde{\varphi}\left(a_{1} t\right) & =\sup _{s \geq 0}\left(a_{1} t s-\widetilde{\varphi}(s)\right)=\sup _{s \geq 0}\left(2 a_{1} t s-\widetilde{\varphi}(2 s)\right) \geq \\
& \geq \sup _{s>0}\left(2 a_{1} t s-c_{1} \widetilde{\varphi}(s)\right)>2 a_{1} \widetilde{\widetilde{\varphi}}(t) .
\end{aligned}
$$

Further,

$$
\varphi\left(c a_{1}^{k} t\right) \geq \widetilde{\widetilde{\varphi}}\left(a_{1}^{k} c t\right) \geq 2^{k} a_{1}^{k} \approx \widetilde{\varphi}(c t) \geq 2^{k} a_{1}^{k} \varphi(t) .
$$

For $2^{k} \geq 2 c$ the latter estimate implies $\varphi(a t) \geq 2 a \varphi(t)$, where $a=c a_{1}^{k}$. 


\section{$\S 2$ 2. A Weak Type One-Weighted Problem in Orlicz Classes for Maximal Functions (the Scalar Case)}

We begin by presenting two results to be used in our further reasoning. The first of them describes the class of those functions $\varphi$ from $\Phi$ for which a strong type inequality is fulfilled in the nonweighted case.

Theorem A. Let $\varphi \in \Phi, \mu E>0$. Then the conditions below are equivalent:

(i) the inequality

$$
\int_{E} \varphi(\mathbf{M} f(x)) d \mu \leq c \int_{E} \varphi(c f(x)) d \mu
$$

holds for an arbitrary $\mu$-measurable function $f$ with the condition $\operatorname{supp} f \subset$ $E$ and with the constant $c$ not depending on $f$;

(ii) $\varphi^{\alpha}$ is quasi-convex for some $\alpha, 0<\alpha<1$.

For $E=X$ the proof of Theorem $\mathrm{A}$ is given in [4]. In the general case the proof is nearly the same and we therefore leave it out.

Theorem B. Let $\varphi \in \Phi$. Then the conditions below are equivalent:

(i) there is a $c_{1}>0$ such that the inequality

$$
\varphi(\lambda) w\{x \in X: \mathbf{M} f(x)>\lambda\} \geq c_{1} \int_{X} \varphi\left(c_{1} f(x)\right) w(x) d \mu
$$

is fulfilled for any $\lambda>0$ and locally summable function $f: X \rightarrow \mathbb{R}^{1}$;

(ii) there are positive constants $\varepsilon$ and $c_{2}$ such that the inequality

$$
\int_{B} \widetilde{\varphi}\left(\varepsilon \frac{\varphi(\lambda)}{\lambda} \frac{w B}{\mu B w(x)}\right) w(x) d \mu \leq c_{2} \varphi(\lambda) w B
$$

is fulfilled for any ball $B$ and positive number $\lambda$;

(iii) there is a positive constant $c_{3}$ such that the inequality

$$
\varphi\left(\frac{1}{\mu B} \int_{B} f(x) d \mu\right) \leq \frac{c_{3}}{w B} \int_{B} \varphi\left(c_{3} f(x)\right) w(x) d \mu .
$$

is fulfilled for any ball $B$ and nonnegative measurable locally summable function $f$ with the condition $\operatorname{supp} f \subset B$.

Theorem $B$ is the particular case of Theorem 5.1 from [16] for $\theta(u) \equiv u$, $\gamma=0, d \beta=w d \mu \otimes \delta_{0}, \eta \equiv 1, \psi(t)=\varphi(t)$ and $\nu(x)=\sigma(x)=w(x)$, where $\delta_{0}$ is the Dirac measure supported at the origin.

Now we shall prove several lemmas on which the proof of Theorem I rests. 
Lemma 2.1. If condition (2.2) is fulfilled for $\varphi$ from $\Phi$ and the weight function $w$, then the function $\varphi$ is quasi-convex and $w \in \mathcal{A}_{s}$ for an arbitrary $s>p(\varphi)$ where $p(\varphi)$ is defined by $(0.6)$.

Proof. We shall show in the first place that in the conditions of the theorem $\varphi$ is quasi-convex. Let $E=\left\{\frac{1}{k}<w(x)<k\right\}$ be such that the set has a positive $\mu$-measure. Choose a ball such that $\mu B \cap E>0$. From (2.2) we have

$$
k \frac{\mu B \cap E}{w B} \widetilde{\varphi}\left(\varepsilon \frac{w B}{\mu B} k \frac{\varphi(\lambda)}{\lambda}\right) \leq c_{1} \varphi(\lambda),
$$

which means that there are positive numbers $\varepsilon_{1}$ and $c_{2}$ such that we have

$$
\widetilde{\varphi}\left(\varepsilon_{1} \frac{\varphi(\lambda)}{\lambda}\right) \leq c_{2} \varphi(\lambda)
$$

for any $\lambda>0$. By virtue of Lemma 1.1 the latter inequality is equivalent to the quasi-convexity of $\varphi$.

The definition of the number $p(\varphi)$ implies that the function $\varphi^{\frac{\alpha}{p(\varphi)}}$ is not quasi-convex for anyone of $\alpha \in(0,1)$. Therefore, according to Lemma 1.2, for an arbitrary $a>1$ there exists a $t>0$ such that

$$
\varphi^{\frac{1}{p(\varphi)}}(a t)<2 a \varphi^{\frac{1}{p(\varphi)}}(t)
$$

or, which is the same thing,

$$
\varphi(a t)<(2 a)^{p(\varphi)} \varphi(t) .
$$

Let $B$ be an arbitrary ball and $E$ be its any $\mu$-measurable subset. Using the Young inequality and condition (2.2), we obtain

$$
\begin{gathered}
w B=\frac{1}{2 c_{2} \varphi(t)} \int_{E} \frac{2 c_{2}}{\varepsilon} t \frac{\mu B}{\mu E} \varepsilon \frac{\varphi(t)}{t} \frac{w B}{\mu B w(x)} w(x) d \mu \leq \\
\leq \frac{1}{2 c_{2} \varphi(t)} \varphi\left(\frac{2 c_{2}}{\varepsilon} t \frac{\mu B}{\mu E}\right) w E+\frac{1}{2 c_{2} \varphi(t)} \int_{E} \widetilde{\varphi}\left(\frac{\varepsilon \varphi(t)}{t} \frac{w B}{\mu B w(x)}\right) w(x) d \mu \leq \\
\leq \frac{1}{2 c_{2} \varphi(t)} \varphi\left(\frac{2 c_{2}}{\varepsilon} t \frac{\mu B}{\mu E}\right) w E+\frac{1}{2} w B
\end{gathered}
$$

from which we conclude that

$$
\frac{w B}{w E} \varphi(t) \leq c_{2} \varphi\left(c_{2} \frac{\mu B}{\mu E} t\right) .
$$

Let $a=c_{2} \frac{\mu B}{\mu E}$ and $t$ be a corresponding number such that (2.4) holds. On substituting this value of $t$ in (2.5), we get

$$
\frac{w B}{w E} \varphi(t) \leq c_{2} \varphi\left(c_{2} \frac{\mu B}{\mu E} t\right) \leq c_{2}\left(c_{2} \frac{\mu B}{\mu E}\right)^{p(\varphi)} \varphi(t)
$$


from which we conclude that

$$
\frac{w B}{w E} \leq c\left(\frac{\mu B}{\mu E}\right)^{p(\varphi)}
$$

This means (see [21]) that $w \in \mathcal{A}_{s}$ for an arbitrary $s>p(\varphi)$ when $p(\varphi)>1$ and $w \in \mathcal{A}_{1}$ when $p(\varphi)=1$.

Lemma 2.2. Let condition (2.2) be fulfilled and $\widetilde{\varphi} \in \Delta_{2}$. If

$$
\psi=u \widetilde{\varphi}\left(\frac{1}{u}\right)
$$

then the function $\psi(t w) \in \mathcal{A}_{\infty}$ uniformly with respect to $t, t>0$.

Proof. Let $B$ be an arbitrary ball and $E$ be its any $\mu$-measurable subset. The convexity of the function $\widetilde{\varphi}$ implies that $\frac{\widetilde{\varphi}(t)}{t}$ increases. Using this fact and the condition $\widetilde{\varphi} \in \Delta_{2}$, from (2.2) we obtain

$$
\int_{B} \tilde{\varphi}\left(\frac{\varphi(\lambda)}{\lambda} \frac{w E}{\mu B w(x)}\right) w(x) d \mu \leq c \varphi(\lambda) w E,
$$

where $c$ does not depend on $\lambda, B$ and $E$.

Setting

$$
\frac{\varphi(\lambda)}{\lambda} \frac{w E}{\mu B}=\frac{1}{t}
$$

we have

$$
\int_{B} \widetilde{\varphi}\left(\frac{1}{t w(x)}\right) t w(x) d \mu \leq \operatorname{ct} \varphi(\lambda) w E .
$$

From the expression for $t$ and the Young inequality we obtain

$$
t \varphi(\lambda) w E \leq \frac{t}{2} \varphi(\lambda) w E+\frac{1}{2} \int_{E} \widetilde{\varphi}\left(2 \frac{\mu B}{\mu E} \frac{1}{t w(x)}\right) t w(x) d \mu .
$$

Hence we conclude that

$$
\int_{B} \widetilde{\varphi}\left(\frac{1}{t w(x)}\right) t w(x) d \mu \leq c \int_{E} \widetilde{\varphi}\left(2 \frac{\mu B}{\mu E} \frac{1}{t w(x)}\right) t w(x) d \mu .
$$

The condition $\widetilde{\varphi} \in \Delta_{2}$ implies that (see [3], Lemma 1.3.2)

$$
\widetilde{\varphi}(a \tau) \leq c_{1} a^{p} \widetilde{\varphi}(\tau)
$$

where the constant $c_{1}$ does not depend on $a>1$ and $\tau>0$. If in the latter inequality we take $a=\frac{\mu B}{\mu E}$ and $\tau=\frac{1}{t w(x)}$, we shall obtain

$$
\widetilde{\psi}\left(\frac{\mu E}{\mu B} t w(x) t w(x)\right) \leq c\left(\frac{\mu B}{\mu E}\right)^{p} \widetilde{\psi}(t w(x)) .
$$


Using (2.9), from the inequality (2.7) we obtain

$$
\int_{B} \psi(t w(x)) d \mu \leq c\left(\frac{\mu B}{\mu E}\right)^{p} \int_{E} \psi(t w(x)) d \mu .
$$

Thus $\psi(t w) \in \mathcal{A}_{\infty}$ uniformly with respect to $t$.

Lemma 2.3. Let $\varphi \in \Phi$ and $\varphi^{\alpha}$ be quasi-convex for some $\alpha, 0<\alpha<1$. If now condition (2.2) is fulfilled, then there is a convex function $\varphi_{0}$ such that $p(\varphi)>p\left(\varphi_{0}\right)>1$ and condition (2.2) with $\varphi$ replaced by $\varphi_{0}$ is fulfilled.

Proof. By Lemma 2.2 the function $\psi(t w) \in \mathcal{A}_{\infty}$ uniformly with respect to $t$. Therefore (see [17], [20]) the reverse Hölder inequality

$$
\left(\frac{1}{\mu B} \int_{B} \psi^{1+\delta}(t w(x)) d \mu\right)^{1+\delta} \leq c\left(\frac{1}{\mu B} \int_{B} \psi(t w(x)) d \mu\right)
$$

holds, where the constant $c$ does not depend on $t$.

We set

$$
\psi_{0}(t)=\frac{\widetilde{\varphi}^{1+\delta}(t)}{t^{\delta}} .
$$

Since the function $\widetilde{\varphi}$ is convex, $\psi_{0}$ will be convex, too. Therefore if $\varphi_{0}=$ $\widetilde{\psi}_{0}$, we shall have $\widetilde{\varphi}_{0}=\widetilde{\psi}_{0}=\psi_{0}$. Moreover, the condition $\widetilde{\varphi} \in \Delta_{2}$ implies $\widetilde{\varphi}_{0} \in \Delta_{2}$. By Lemma 1.2 hence it follows that $p\left(\varphi_{0}\right)>1$.

Substituting $t=\frac{\lambda}{\varphi_{0}(\lambda)} \frac{\mu B}{w B}$ into (2.10) and making use of (2.11), we obtain

$$
\begin{aligned}
& \left(\frac{1}{\varphi_{0}(\lambda) w B} \int_{B} \widetilde{\varphi}_{0}\left(\frac{\varphi_{0}(\lambda)}{\lambda} \frac{w B}{\mu B w(x)}\right) w(x) d \mu\right)^{\frac{1}{1+\delta}} \leq \\
& \leq c \lambda^{\frac{\delta}{1+\delta}}\left(\varphi_{0}(\lambda) w B\right)^{-1} \int_{B} \widetilde{\varphi}\left(\frac{\varphi_{0}(\lambda)}{\lambda} \frac{w B}{\mu B w(x)}\right) w(x) d \mu .
\end{aligned}
$$

Let $s$ be such that for a given $\lambda$

$$
\frac{\varphi_{0}(\lambda)}{\lambda}=\frac{\varphi(s)}{s}
$$

Then by virtue of (1.5) and the condition $\widetilde{\varphi} \in \Delta_{2}$ we have

$$
\begin{gathered}
\varphi(s) \leq \widetilde{\varphi}\left(2 \frac{\varphi(s)}{s}\right) \leq c \widetilde{\varphi}\left(\frac{\varphi_{0}(\lambda)}{\lambda}\right)= \\
=c\left(\widetilde{\varphi}_{0}\left(\frac{\varphi_{0}(\lambda)}{\lambda}\right)\right)^{\frac{1}{1+\delta}}\left(\frac{\varphi_{0}(\lambda)}{\lambda}\right)^{\frac{\delta}{1+\delta}} \leq c \varphi_{0}(\lambda) \lambda^{-\frac{\delta}{1+\delta}} .
\end{gathered}
$$


Therefore

$$
\frac{\lambda^{\frac{\delta}{1+\delta}}}{\varphi_{0}(\lambda)} \leq c \frac{1}{\varphi(s)} .
$$

Now from (2.13) and (2.12) we conclude that

$$
\frac{1}{\varphi_{0}(\lambda) w B} \int_{B} \widetilde{\varphi}_{0}\left(\frac{\varphi_{0}(\lambda)}{\lambda} \frac{w B}{\mu B w(x)}\right) w(x) d \mu \leq c .
$$

Thus (2.2) holds, where $\varphi$ is replaced by the convex function $\varphi_{0}$. Now it remains for us to show that $p(\varphi)>p\left(\varphi_{0}\right)$. First, we shall prove that there are constants $c_{1}$ and $c_{2}$ such that

$$
c_{1} t^{\frac{\delta}{1+\delta}} \varphi\left(c_{1} t^{\frac{1}{1+\delta}}\right) \leq \varphi_{0}(t) \leq c_{2} t^{\frac{\delta}{1+\delta}} \varphi\left(c_{2} t^{\frac{1}{1+\delta}}\right) .
$$

Using (1.5), (1.6) and the Young inequality, on the one hand, we have

$$
\begin{aligned}
\varphi_{0}(t) & =t^{\frac{\delta}{1+\delta}} \varepsilon \frac{\varphi_{0}(t)}{t} \frac{1}{\varepsilon} t^{\frac{1}{1+\delta}} \leq t^{\frac{\delta}{1+\delta}} \widetilde{\varphi}\left(\varepsilon \frac{\varphi_{0}(t)}{t}\right)+t^{\frac{\delta}{1+\delta}} \varphi\left(\frac{1}{\varepsilon} t^{\frac{1}{1+\delta}}\right)= \\
& =\widetilde{\varphi}_{0}^{\frac{1}{1+\delta}}\left(\varepsilon \frac{\varphi_{0}(t)}{t}\right)\left(\varepsilon \varphi_{0}(t)\right)^{\frac{\delta}{1+\delta}}+t^{\frac{\delta}{1+\delta}} \varphi\left(\frac{1}{\varepsilon} t^{\frac{1}{1+\delta}}\right) \leq \\
& \leq \varepsilon^{\frac{\delta}{1+\delta}} \varphi_{0}^{\frac{1}{1+\delta}}(t)\left(\varphi_{0}(t)\right)^{\frac{\delta}{1+\delta}}+t^{\frac{\delta}{1+\delta}} \varphi\left(\frac{1}{\varepsilon} t^{\frac{1}{1+\delta}}\right) \leq \\
& \leq \varepsilon^{\frac{\delta}{1+\delta}} \varphi_{0}(t)+t^{\frac{\delta}{1+\delta}} \varphi\left(\frac{1}{\varepsilon} t^{\frac{1}{1+\delta}}\right) .
\end{aligned}
$$

Hence we conclude that

$$
\varphi_{0}(t) \leq c_{2} t^{\frac{\delta}{1+\delta}} \varphi\left(c_{2} t^{\frac{1}{1+\delta}}\right)
$$

On the other hand,

$$
\begin{aligned}
& t^{\frac{\delta}{1+\delta}} \varphi\left(t^{\frac{1}{1+\delta}}\right)=\frac{\varepsilon^{\delta}}{2} \varepsilon \frac{\varphi\left(t^{\frac{1}{1+\delta}}\right)}{t^{\frac{1}{1+\delta}}} \frac{2 t}{\varepsilon^{1+\delta}} \leq \frac{\varepsilon^{\delta}}{2} \varphi_{0}\left(\frac{2 t}{\varepsilon^{1+\delta}}\right)+\frac{\varepsilon^{\delta}}{2} \widetilde{\varphi}_{0}\left(\frac{\varepsilon \varphi\left(t^{\frac{1}{1+\delta}}\right)}{t^{\frac{1}{1+\delta}}}\right)= \\
& =\frac{\varepsilon^{\delta}}{2} \varphi_{0}\left(\frac{2 t}{\varepsilon^{1+\delta}}\right)+\frac{\varepsilon^{\delta}}{2}\left(\varepsilon \frac{\varphi\left(t^{\frac{1}{1+\delta}}\right)}{t^{\frac{1}{1+\delta}}}\right)^{-\delta} \widetilde{\varphi}^{1+\delta}\left(\varepsilon \frac{\varphi\left(t^{\frac{1}{1+\delta}}\right)}{t^{\frac{1}{1+\delta}}}\right) \leq \\
& \leq \frac{\varepsilon^{\delta}}{2} \varphi_{0}\left(\frac{2}{\varepsilon^{1+\delta}} t\right)+\frac{1}{2} \varphi_{0}\left(t^{\frac{1}{1+\delta}}\right) t^{\frac{\delta}{1+\delta}} .
\end{aligned}
$$

This implies

$$
t^{\frac{\delta}{1+\delta}} \varphi\left(t^{\frac{1}{1+\delta}}\right) \leq \varepsilon^{\delta} \varphi_{0}\left(\frac{2}{\varepsilon^{1+\delta}} t\right)
$$

Inequality (2.15) is therefore proved. From the definition of $p\left(\varphi_{0}\right)$ the function $\varphi_{0}^{\frac{1}{p\left(\varphi_{0}\right)-\varepsilon}}$ is quasi-convex for an arbitrary sufficiently small $\varepsilon>0$. By Lemma 1.1 this is equivalent to the fact that the function $t^{-1} \varphi^{\frac{1}{p\left(\varphi_{0}\right)-\varepsilon}}(t)$ almost increases. On account of (2.15) this means that the function 
$t^{\varepsilon-p\left(\varphi_{0}\right)} \varphi\left(t^{\frac{1}{1+\delta}}\right) t^{\frac{\delta}{1+\delta}}$ is almost increasing. Therefore the function $\varphi(u) u^{-\left(\left(p\left(\varphi_{0}\right)-\varepsilon\right)(1+\delta)-\delta\right)}$ almost increases. The latter conclusion is equivalent to the fact that the function $\varphi^{\frac{1}{(1+\delta)\left(p\left(\varphi_{0}\right)-\varepsilon\right)-\delta}}$ is quasi-convex. From the definition of $p(\varphi)$ we have $p(\varphi)>(1+\delta)\left(p\left(\varphi_{0}\right)-\varepsilon\right)-\delta$ for a sufficiently small $\varepsilon$. Since $p\left(\varphi_{0}\right)>1$, we conclude that $p(\varphi)>p\left(\varphi_{0}\right)$.

Proof of Theorem I. First, we shall prove that (ii) $\Rightarrow$ (i). By virtue of the $\mathcal{A}_{p^{-}}$ condition there is a $p_{1}<p(\varphi)$ such that $w \in \mathcal{A}_{p_{1}}$. On the other hand, the definition of $p(\varphi)$ implies that the function $\varphi^{\frac{1}{p_{1}}}$ is quasi-convex. Applying the definition of quasi-convexity, the Jensen inequality and the fact that the operator $\mathbf{M}: f \rightarrow \mathbf{M} f$ is bounded in $L_{w}^{p_{1}}(X)$ for $w \in \mathcal{A}_{p_{1}}$ (see [20]), we obtain

$$
\begin{gathered}
\int_{X} \varphi(\mathbf{M} f(x)) w(x) d \mu=\int_{X}\left[\varphi^{\frac{1}{p_{1}}}(\mathbf{M} f(x))\right]^{p_{1}} w(x) d x \leq \\
\leq c \int_{X}\left(\mathbf{M}\left(\varphi^{\frac{1}{p_{1}}}(c f(x))\right)\right)^{p_{1}} w(x) d x \leq c_{1} \int_{X} \varphi\left(c_{1} f(x) w(x) d x .\right.
\end{gathered}
$$

Next we shall show that (i) $\Rightarrow$ (ii). Choose $k>0$ such that the set $E=$ $\left\{k^{-1} \leq w(x) \leq k\right\}$ have a positive measure. Then from the condition (i) it follows that

$$
\int_{E} \varphi(\mathbf{M} f(x)) d \mu \leq c k^{2} \int_{E} \varphi(c f(x)) d \mu
$$

for an arbitrary $f$ provided that supp $f \subset E$. By Theorem A hence we conclude that $\varphi^{\alpha}$ is quasi-convex for some $\alpha, 0<\alpha<1$. Now let us prove that $w \in \mathcal{A}_{p(\varphi)}$. The condition (i) implies that inequality (2.2) is fulfilled. Applying Lemma 2.3, we arrive at the existence of a convex function $\varphi_{0}$ such that

$$
\int_{B} \widetilde{\varphi}\left(\varepsilon \frac{\varphi_{0}(\lambda)}{\lambda} \frac{w B}{\mu B w(x)}\right) w(x) d \mu \leq c_{2} \varphi_{0}(\lambda) w B,
$$

where the constant $c_{2}$ does not depend on $\lambda$ and the ball $B$ and, besides, $p(\varphi)>p\left(\varphi_{0}\right)>1$. But in that case, according to Lemma 2.1, the function $w \in \mathcal{A}_{s}$ for any $s>p\left(\varphi_{0}\right)$ and therefore $w \in \mathcal{A}_{p(\varphi)}$.

Finally, we wish to make some useful remarks.

Proposition 2.4. Either of conditions (2.1) and (2.2) is equivalent to the fact that the function $\varphi$ is quasi-convex and $w \in \mathcal{A}_{p(\varphi)}$.

Proof. The fact that the condition $w \in \mathcal{A}_{p(\varphi)}$ implies (2.2) (and, accordingly, 2.1) can be proved directly.

Let $w \in \mathcal{A}_{p(\varphi)}$ and $p(\varphi)>1$. Then there is a $p_{2}<p(\varphi)$ such that $w \in \mathcal{A}_{p_{2}}$. The definition of $p(\varphi)$ implies the existence of a $p_{1}$ such that $p_{2}<$ 
$p_{1}<p(\varphi)$ and the function $\varphi^{\frac{1}{p_{1}}}$ is quasi-convex. Therefore by Corollary 1.1 we have $s^{p_{1}} \varphi(t) \leq \varphi(c s t), s \geq 1$. Hence for $a>1$ we obtain

$$
\begin{aligned}
\widetilde{\varphi}(a t) & =\sup _{s>0}(s a t-\varphi(s))=\sup _{s>0}\left(a^{\frac{p_{1}}{p_{1}-1}} t c s-\varphi\left(a^{\frac{1}{p_{1}-1}} c s\right) \leq\right. \\
& \leq \sup _{s>0}\left(a^{\frac{p_{1}}{p_{1}-1}} c t s-a^{\frac{p_{1}}{p_{1}-1}} \varphi(s)\right)=a^{\frac{p_{1}}{p_{1}-1}} \widetilde{\varphi}(c t) .
\end{aligned}
$$

From the latter estimate, inequality (1.5) and the condition $w \in \mathcal{A}_{p_{1}}$ we derive

$$
\begin{gathered}
\int_{\left\{x: \frac{w B}{\mu B w(x)}>1\right\}} \widetilde{\varphi}\left(\varepsilon \lambda \frac{w B}{\mu B w(x)}\right) w(x) d x \leq \\
\leq \widetilde{\varphi}(c \varepsilon \lambda) \int_{B}\left(\frac{w B}{\mu B w(x)}\right)^{\frac{p_{1}}{p_{1}-1}} w(x) d \mu \leq c \widetilde{\varphi}(\lambda) w B .
\end{gathered}
$$

Thus

$$
\int_{B} \widetilde{\varphi}\left(\varepsilon \lambda \frac{w B}{\mu B w(x)}\right) w(x) d x \leq \widetilde{\varphi}(\varepsilon \lambda) w B+c \widetilde{\varphi}(\lambda) w B \leq c_{1} \widetilde{\varphi}(\lambda) w B .
$$

Let now $p(\varphi)=1$. Then the function $\frac{w B}{\mu B w(x)}$ is bounded on $B$ by a constant independent of $B$ and we have (2.16).

Further, if in inequality (2.16) we replace $\lambda$ by $\varepsilon_{0} \frac{\varphi(\lambda)}{\lambda}$ where $\varepsilon_{0}$ is the respective constant from (1.3) and in the right-hand side use the abovementioned inequality, then we shall obtain (2.2).

Proposition 2.5. Let $\varphi$ be quasi-convex. The conditions below are equivalent:

(i) there are constants $\varepsilon_{1}$ and $c_{1}$ such that

$$
\varphi\left(\frac{\varepsilon_{1}}{\lambda \mu B} \int_{B} \widetilde{\varphi}\left(\frac{\lambda}{w(x)}\right) w(x) d \mu\right) w B \leq c_{1} \int_{B} \widetilde{\varphi}\left(\frac{\lambda}{w(x)}\right) w(x) d \mu
$$

for any ball $B$ and number $\lambda>0$;

(ii) there are constants $\varepsilon_{2}$ and $c_{2}$ such that

$$
\int_{B} \widetilde{\varphi}\left(\varepsilon_{2} \frac{\lambda w B}{w(x) \mu B}\right) w(x) d \mu \leq c_{2} \widetilde{\varphi}(\lambda) w B
$$

for any ball $B$ and number $\lambda>0$;

(iii) $w \in \mathcal{A}_{p(\varphi)}$. 
Proof. It is easy to show that (i) $\Rightarrow($ ii). To this effect in (2.17) it is sufficient to replace $\lambda$ by $\frac{\lambda w B}{2 c_{1} \mu B}$. Then $(2.17)$ can be rewritten as

$$
\frac{2 \varphi\left(\frac{2 c_{1}}{\lambda w B} \int_{B} \widetilde{\varphi}\left(\frac{1}{2 c_{1}} \frac{\lambda w B}{\mu B}\right) w(x) d \mu\right)}{\frac{2 c_{1}}{\lambda w B} \int_{B} \widetilde{\varphi}\left(\frac{1}{2 c_{1}} \frac{\lambda w B}{\mu B}\right) w(x) d \mu} \leq \lambda .
$$

Taking into account that $\frac{\widetilde{\varphi}(t)}{t}$ increases and using inequality (1.5), we conclude from (2.19) that (2.18) is valid.

The implication (ii) $\Rightarrow$ (iii) is obtained as follows. In Proposition 2.4 it was actually proved that (ii) $\Rightarrow(2.2)$. By Lemma 2.4 it follows from (2.2) that $w \in \mathcal{A}_{p(\varphi)}$. The reverse statement was shown in proving Proposition 2.4 .

Now we proceed to proving Theorem III. The proof will be based on the following propositions.

Proposition 2.6. Let $\varphi \in \Phi$. Then the statements below are equivalent:

(i) there is a constant $c$ such that the inequality

$$
\int_{\{x: \mathbf{M} f(x)>\lambda\}} \varphi\left(\frac{\lambda}{w(x)}\right) w(x) d \mu \leq c \int_{X} \varphi\left(c \frac{f(x)}{w(x)}\right) w(x) d \mu
$$

is fulfilled for any $\mu$-measurable function $f: X \rightarrow \mathbb{R}^{1}$ and an arbitrary $\lambda>0$;

(ii) the function $\varphi$ is quasi-convex and there are positive constants $\varepsilon>0$ and $c_{1}>0$ such that

$$
\widetilde{\varphi}\left(\frac{\varepsilon}{\lambda \mu B} \int_{B} \varphi\left(\frac{\lambda}{w(x)}\right) w(x) d \mu\right) w B \leq c_{1} \int_{B} \varphi\left(\frac{\lambda}{w(x)}\right) w(x) d \mu .
$$

Since the proof of this proposition repeats that of Theorem 5.1 from [16], we leave it out.

If in Proposition 2.6 we replace $\varphi$ by $\widetilde{\varphi}$ and take into account that $\widetilde{\varphi} \sim \varphi$ for a quasi-convex function $\varphi$ (see Lemma 1.1), then by Proposition 2.5 we conclude that the following proposition is valid.

Proposition 2.7. Let $\varphi \in \Phi$. The conditions below are equivalent: 
(i) the function $\varphi$ is quasi-convex and there is a constant $c_{1}>0$ such that the inequality

$$
\int_{\{x: \mathbf{M} f(x)>\lambda\}} \widetilde{\varphi}\left(\frac{\lambda}{w(x)}\right) w(x) d \mu \leq c_{1} \int_{X} \widetilde{\varphi}\left(c_{1} \frac{f(x)}{w(x)}\right) w(x) d \mu
$$

is fulfilled for any $\lambda>0$ and $\mu$-measurable function $f: X \rightarrow \mathbb{R}^{1}$;

(ii) there is a constant $c_{2}>0$ such that the inequality

$$
\varphi(\lambda) \int_{\{x: \mathbf{M} f(x)>\lambda\}} w(x) d \mu \leq c_{2} \int_{X} \varphi\left(c_{2} f(x)\right) w(x) d \mu
$$

is fulfilled for an arbitrary $\lambda>0$;

(iii) the function $\varphi$ is quasi-convex and there are positive numbers $\varepsilon$ and $c_{3}$ such that

$$
\varphi\left(\frac{\varepsilon}{\lambda \mu B} \int_{B} \widetilde{\varphi}\left(\frac{\lambda}{w(x)}\right) w(x) d \mu\right) w B \leq c_{3} \int_{B} \widetilde{\varphi}\left(\frac{\lambda}{w(x)}\right) w(x) d \mu
$$

is fulfilled for any $\lambda>0$ and an arbitrary ball $B$;

(iv) there are positive numbers $\varepsilon$ and $c_{4}$ such that the inequality

$$
\int_{B} \widetilde{\varphi}\left(\varepsilon \frac{\varphi(\lambda)}{\lambda} \frac{w B}{w(x) \mu B}\right) w(x) d \mu \leq c_{4} \varphi(\lambda) w B
$$

is fulfilled for any $\lambda>0$ and ball $B$;

(v) the function $\varphi$ is quasi-convex and $w \in \mathcal{A}_{p(\varphi)}$.

Proof of Theorem III. First, we shall prove the implication (i) $\Rightarrow$ (iii). From the condition (i) we obtain a weak type inequality. Moreover, the same condition implies that $\varphi^{\alpha}$ is quasi-convex. Indeed, the condition (i) implies that the inequality

$$
\int_{E} \varphi(\mathbf{M} f(x)) d \mu \leq c \int_{E} \varphi(c f(x)) d \mu
$$

is fulfilled on the set $E=\left\{\frac{1}{k}<w(x)<k\right\}$ where $k$ is a number such that $\mu E>0$. Therefore on account of Theorem A the function $\varphi^{\alpha}$ is quasiconvex for some $\alpha, 0<\alpha<1$. Further by Lemma 1.2 the quasi-convexity of $\varphi^{\alpha}(0<\alpha<1)$ implies $\widetilde{\varphi} \in \Delta_{2}$. Now by Proposition 2.6 from (i) we conclude that (iii) is valid.

The implication (iii) $\Rightarrow$ (iv) follows from Proposition 2.5. We shall prove the validity of the implication (iv) $\Rightarrow(\mathrm{i})$. By virtue of Lemma 2.1 the condition (iv) implies $w \in \mathcal{A}_{\infty}$. Now we shall use the method developed in [25]. 
Let $B_{j}^{k}$ and $E_{j}^{k}(j \in N, k \in Z)$ be respectively balls and sets from Lemma 2 of [2]. We set

$$
m_{B_{j}^{k}}(f)=\frac{1}{\mu B_{j}^{k}} \int_{B_{j}^{k}} f(y) d \mu .
$$

Applying the above-mentioned lemma, we obtain

$$
\int_{X} \varphi\left(\frac{\mathbf{M} f(x)}{w(x)}\right) w(x) d \mu \leq \sum_{k, j} \int_{E_{j}^{k}} \varphi\left(\frac{b^{2} m_{B_{j}^{k}}(f)}{w(x)}\right) w(x) d \mu .
$$

Now in the condition (iv) we set

$$
\lambda=\frac{1}{w B_{j}^{k}} \int_{B_{j}^{k}}|f(y)| d \mu
$$

and use the resulting inequality to estimate the right-hand side of (2.20). This leads us to the estimates

$$
\begin{gathered}
\int_{X} \varphi\left(\frac{\mathbf{M} f(x)}{w(x)}\right) w(x) d \mu \leq c \sum_{k, j} \varphi\left(\frac{b^{2} \int_{B_{j}^{k}}|f(y)| d \mu}{w B_{j}^{k}}\right) w B_{j}^{k} \leq \\
\leq c \sum_{k, j} \varphi\left(\frac{b^{2}}{w B_{j}^{k}} \int_{B_{j}^{k}} \frac{|f(x)|}{w(x)} w(x) d \mu\right) w E_{j}^{k} .
\end{gathered}
$$

We set

$$
\mathbf{M}_{w} f(x)=\sup _{B \ni x} \frac{1}{w B} \int_{B}|f(y)| w(y) d \mu,
$$

which implies that

$$
\begin{gathered}
\int_{X} \varphi\left(\frac{\mathbf{M} f(x)}{w(x)}\right) w(x) d \mu \leq c \sum_{k, j} \int_{E_{j}^{k}} \varphi\left(\mathbf{M}_{w}\left(\frac{b^{2} f(x)}{w(x)}\right)\right) w(x) d \mu \leq \\
\leq c \int_{X} \varphi\left(b^{2} \mathbf{M}_{w}\left(\frac{f(x)}{w(x)}\right)\right) w(x) d \mu .
\end{gathered}
$$

On the other hand, the function $\varphi^{\alpha}$ is quasi-convex for some $\alpha \in(0,1)$ and $w \in \mathcal{A}_{\infty}$. The latter condition implies that $w$ satisfies the doubling condition. Therefore we are able to apply Theorem A to the right-hand side of the above inequality. As a result, we conclude that

$$
\int_{X} \varphi\left(\frac{\mathbf{M} f(x)}{w(x)}\right) w(x) d \mu \leq c \int_{X} \varphi\left(c \frac{f(x)}{w(x)}\right) w(x) d \mu .
$$




\section{$\S 3$. Criterion of a Strong Type One-Weighted Inequality for} Vector-Valued Functions. The proof of Theorem II

Let $f=\left(f_{1}, f_{2}, \ldots, f_{n}, \ldots\right)$ where $f_{j}: X \rightarrow \mathbb{R}^{1}$ are $\mu$-measurable locally summable functions for each $i=1,2, \ldots, n$. For $\theta, 1<\theta<\infty$, and $x \in X$ we set

$$
\|f(x)\|_{\theta}=\left(\sum_{j=1}^{\infty}\left|f_{j}(x)\right|^{\theta}\right)^{\frac{1}{\theta}} .
$$

Let $\mathbf{M} f=\left(\mathbf{M} f_{1}, \mathbf{M} f_{2}, \ldots, \mathbf{M} f_{n}, \ldots\right)$.

The proof of Theorem II will be based on some auxiliary results to be discussed below.

Theorem 3.1. Let $1<p, \theta<\infty$. Then the following conditions are equivalent:

(i) there is a constant $c>0$ such that the inequality

$$
\int_{X}\|\mathbf{M} f(x)\|_{\theta}^{p} w(x) d \mu \leq c \int_{X}\|f(x)\|_{\theta}^{p} w(x) d \mu
$$

is fulfilled for any vector-function $f$;

(ii) $w \in \mathcal{A}_{p}(X)$.

To prove the theorem we need the following lemmas:

Lemma A ([17], Lemma 2). Let $\mathcal{F}$ be a family $\{B(x, r)\}$ of balls with bounded radii. Then there is a countable subfamily $\left\{B\left(x_{i}, r_{i}\right)\right\}$ consisting of pairwise disjoint balls such that each ball in $\mathcal{F}$ is contained in one of the balls $B\left(x_{i}\right.$, ar $\left.r_{i}\right)$ where $a=3 a_{1}^{2}+2 a_{0} a_{1}$.

Lemma 3.1. Let $1<p<\infty, f: X \rightarrow \mathbb{R}^{1}, \varphi: X \rightarrow \mathbb{R}^{1}$ be non-negative measurable functions. Then there is a constant $c>0$, not depending on $f$ and $\varphi$, such that

$$
\int_{X}(\mathbf{M} f(x))^{p} \varphi(x) d \mu \leq c \int_{X} f^{p}(x) \mathbf{M} \varphi(x) d \mu .
$$

Proof. This lemma is well-known for classical maximal functions and so we give its proof just for the sake of completeness of our discussion.

As can be easily verified, for any nonnegative locally summable function $\varphi$ we have the estimate

$$
\frac{1}{\mu B} \int_{B} \varphi(x) d \mu\left(\frac{1}{\mu B} \int_{B}(\mathbf{M} \varphi(x))^{-\frac{1}{p-1}} d \mu\right)^{p-1} \leq c,
$$

where $c$ does not depend on the ball $B$. 
Further, let $\lambda>0$ and $B_{0}$ be a fixed ball in $X$. We set

$$
H^{\lambda}=\{x \in X: \mathbf{M} f(x)>\lambda\} \cap B_{0} .
$$

Obviously, for an arbitrary point $x \in H^{\lambda}$ there is a ball $B\left(x, r_{x}\right)$ such that

$$
\frac{1}{\mu B\left(x, r_{x}\right)} \int_{B\left(x, r_{x}\right)} f(y) d y>\lambda .
$$

According to Lemma A, from the family $\left\{B\left(x, r_{x}\right)\right\}$ we can choose pairwise disjoint balls $B\left(x_{j}, a r_{j}\right)$ such that each chosen ball will be contained in one of the balls $B\left(x_{j}, a r_{j}\right)$ where $a$ is the absolute constant. Applying the Hölder inequality, the doubling property of the measure $\mu$ and (3.2), we obtain

$$
\begin{gathered}
\int_{H^{\lambda}} \varphi(x) d \mu \leq \sum_{j=1}^{\infty} \int_{B\left(x_{j}, a r_{j}\right)} \varphi(x) d \mu \leq \lambda^{-p} \sum_{j=1}^{\infty} \frac{1}{\mu B_{j}} \int_{B\left(x_{j}, a r_{j}\right)} \varphi(x) d \mu \times \\
\times\left(\int_{B\left(x_{j}, r_{j}\right)} f^{p}(x) \mathbf{M} \varphi(x) d \mu\right)\left(\frac{1}{\mu B_{j}} \int_{B_{j}}(\mathbf{M} \varphi(x))^{-\frac{1}{p-1}} d \mu\right)^{p-1} \leq \\
\leq c \lambda^{-p} \sum_{j=1}^{\infty} \int_{B\left(x_{j}, r_{j}\right)} f^{p}(x) \mathbf{M} \varphi(x) d \mu \leq c \lambda^{-p} \int_{X} f^{p}(x) \mathbf{M} \varphi(x) d \mu .
\end{gathered}
$$

Now to complete the proof we only have to apply Marcinkiewicz' interpolation theorem.

Proof of Theorem 3.1. Let $1<p<\theta<\infty$ and $w \in \mathcal{A}_{p}(X)$. Since inequality (0.1) is fulfilled for $\varphi(u)=u^{p}, 1<p<\infty$, and $w \in \mathcal{A}_{p}$ (see [20]), we have

$$
\int_{X}\|\mathbf{M} f(x)\|_{p}^{p} w(x) d x \leq c_{1} \int_{X}\|f(x)\|_{p}^{p} w(x) d x
$$

and also

$$
\begin{gathered}
\int_{X}\left(\sup _{i} \mathbf{M} f_{i}(x)\right)^{p} w(x) d x \leq \int_{X}\left(\mathbf{M}\left(\sup _{i} f_{i}(x)\right)\right)^{p} w(x) d x \leq \\
\leq c_{2} \int_{X}\left(\sup f_{i}(x)\right)^{p} w(x) d x .
\end{gathered}
$$

If we apply an interpolation theorem of the Marcinkiewicz type (see, for example, [24]), (3.1) will hold for an arbitrary $\theta, 1<p<\theta<\infty$.

Next let $1<\theta<p<\infty$. By virtue of the property of the class $\mathcal{A}_{p}(X)$ there is a number $\theta_{0}<p$ such that $w \in \mathcal{A}_{p / \theta}$ for an arbitrary $\theta, 1<\theta \leq$ 
$\theta_{0}<p$. It will be now shown that (3.1) holds for an arbitrary $\theta$ provided that $1<\theta \leq \theta_{0}<p$.

We have

$$
\left(\int_{X}\|\mathbf{M} f(x)\|_{\theta}^{p} w(x) d \mu\right)^{\theta / p}=\sup \left|\int_{X}\|\mathbf{M} f(x)\|_{\theta}^{\theta} \varphi(x) d \mu\right|,
$$

where the least upper bound is taken with respect to all functions $\varphi: X \rightarrow$ $\mathbb{R}^{1}$ for which

$$
\int_{X}|\varphi(x)|^{\frac{p}{p-\theta}}(w(x))^{-\frac{\theta}{p-\theta}} d \mu \leq 1 .
$$

By virtue of (3.2) we obtain

$$
\begin{gathered}
\int_{X}\left(\sum_{i=1}^{\infty} \mathbf{M}^{\theta} f_{i}(x)\right)|\varphi(x)| d \mu=\sum_{i=1}^{\infty} \int_{X} \mathbf{M}^{\theta} f_{i}(x)|\varphi(x)| d \mu \leq \\
\leq c \sum_{i=1}^{\infty} \int_{X}\left|f_{i}(x)\right|^{\theta} \mathbf{M} \varphi(x) d \mu=c \int_{X}\|f(x)\|_{\theta}^{\theta} \mathbf{M} \varphi(x) d \mu .
\end{gathered}
$$

Applying the Hölder inequality to the latter expression, we have

$$
\begin{gathered}
\int_{X}\left(\sum_{i=1}^{\infty} \mathbf{M}^{\theta} f_{i}(x)\right)|\varphi(x)| d \mu \leq c\left(\int_{X}\left(\sum_{i=1}^{\infty}\left|f_{i}(x)\right|^{\theta}\right)^{p / \theta} w(x) \mid d \mu\right)^{\theta / p} \times \\
\times\left(\int_{X}(\mathbf{M} \varphi(x))^{\frac{p}{p-\theta}} w^{-\frac{\theta}{p-\theta}}(x) d \mu\right)^{\frac{p-\theta}{p}} .
\end{gathered}
$$

The fact $w \in \mathcal{A}_{p / \theta}$ implies $w^{-\frac{\theta}{p-\theta}} \in \mathcal{A}_{\frac{p}{p-\theta}}$. Taking into account (3.3), we estimate the second multiplier in the right-hand side of (3.4) as follows:

$$
\left(\int_{X}\|\mathbf{M} f(x)\|_{\theta}^{p} w(x) d \mu\right)^{\theta / p} \leq \int_{X}\|\mathbf{M} f(x)\|_{\theta}^{\theta}|\varphi(x)| d \mu \leq c\left(\int_{X}\|f(x)\|_{\theta}^{p} w(x) d \mu\right)^{\theta / p}
$$

provided that $1<\theta \leq \theta_{0}$.

Now let us show that (3.1) holds for $\theta_{0}<\theta<p$ as well. Consider two pairs of numbers, $\left(p, \theta_{0}\right)$ and $(p, p)$. By virtue of the above reasoning and the well-known result in the scalar case we have the inequalities

$$
\int_{X}\|\mathbf{M} f(x)\|_{\theta_{0}}^{p} w(x) d \mu \leq c_{1} \int_{X}\|f(x)\|_{\theta_{0}}^{p} w(x) d \mu
$$


and

$$
\int_{X}\|\mathbf{M} f(x)\|_{p}^{p} w(x) d \mu \leq c_{2} \int_{X}\|f(x)\|_{p}^{p} w(x) d \mu .
$$

The proof is completed by applying Marcinkiewicz' interpolation theorem.

Theorem 3.2. Let $\varphi \in \Phi$ and $1<\theta<\infty$. Then the following conditions are equivalent:

(i) there exists a constant $c>0$ such that

$$
\begin{gathered}
\varphi(\lambda) w\left\{x \in X:\left(\sum_{i=1}^{\infty}\left(\mathbf{M} \varphi_{i}(x)\right)^{\theta}\right)^{1 / \theta}>\lambda\right\} \leq \\
\leq c \int_{X} \varphi\left(c\left(\sum_{i=1}^{\infty}\left|f_{i}(x)\right|^{\theta}\right)^{1 / \theta}\right) w(x) d \mu
\end{gathered}
$$

for all $\lambda>0$ and vector-functions $f$;

(ii) the function $\varphi$ is quasi-convex and $\varphi \in \Delta_{2}$.

Proof. The quasi-convexity follows from (3.5) by virtue of Lemma 2.1. We shall prove that $\varphi \in \Delta_{2}$.

Let $x_{0} \in X$ and $\mu\{x\}>0$. We set $r_{0}=1$ and

$$
r_{k}=\sup \left\{r: \mu B\left(x_{0}, r\right)<\frac{1}{2 b} \mu B\left(x_{0}, r_{k-1}\right)\right\}, \quad k=1,2, \ldots,
$$

where the constant $b$ is taken from the doubling condition of the measure $\mu$. Obviously, by the definition of numbers $r_{k}$ we shall have

$$
\begin{gathered}
\mu B\left(x_{0}, r_{k}\right) \backslash B\left(x_{0}, r_{k+1}\right)=\mu B\left(x_{0}, r_{k}\right)-\mu B\left(x_{0}, r_{k+1}\right) \geq \mu B\left(x_{0}, r_{k}\right)- \\
-b \mu B\left(x_{0}, \frac{1}{2} r_{k+1}\right) \geq \mu B\left(x_{0}, r_{k}\right)-\frac{1}{2} \mu B\left(x_{0}, r_{k}\right)=\frac{1}{2} \mu B\left(x_{0}, r_{k}\right) .
\end{gathered}
$$

Therefore

$$
\mu B\left(x_{0}, r_{k}\right) \backslash B\left(x_{0}, r_{k+1}\right) \geq \frac{1}{2} \mu B\left(x_{0}, r_{k}\right) .
$$

Let us define the vector-function $f=\left(f_{1}, \ldots, f_{n}, \ldots\right)$ where

$$
f_{j}(x)=\frac{t}{c} \chi_{B\left(x_{0}, r_{j}\right) \backslash B\left(x_{0}, r_{j+1}\right)}(x),
$$

with the constant $c$ taken from the condition (i).

Obviously,

$$
\left(\sum_{j=1}^{\infty}\left|f_{j}(x)\right|^{\theta}\right)^{1 / \theta}=\frac{t}{c} \mu B\left(x_{0}, r_{1}\right)
$$


At the same time, for any $x \in B\left(x_{0}, r_{j}\right),(j=1,2, \ldots)$, we have on account of (3.6)

$$
\mathbf{M} f_{j}(x) \geq \frac{t}{c} \frac{\mu B\left(x_{0}, r_{j}\right) \backslash B\left(x_{0}, r_{j+1}\right)}{\mu B\left(x_{0}, r_{j}\right)} \geq \frac{t}{2 c} .
$$

Now let $k>4 c$. Then it is obvious that

$$
\left(\sum_{j=1}^{\infty}\left(\mathbf{M} f_{j}(x)\right)^{\theta}\right)^{1 / \theta} \geq \frac{k t}{2 c}>2 t
$$

for an arbitrary $x \in B\left(x_{0}, r_{k}\right)$.

Next set $\lambda=2 t$ in (3.5). By (3.7) we obtain the estimate

$$
\varphi(2 t) w B\left(x_{0}, r_{k}\right) \leq c \varphi(t) w B\left(x_{0}, r_{1}\right) .
$$

Therefore $\varphi \in \Delta_{2}$.

The implication (ii) $\Rightarrow$ (i) can be proved by the arguments used in proving Theorem 1.3.1 from [3].

Proof of Theorem II. The necessary condition for the function $\varphi^{\alpha}$ to be quasi-convex for some $\alpha, 0<\alpha<1$, and $w \in \mathcal{A}_{p(\varphi)}$ follows from the scalar case (Theorem I).

Assume that these conditions are fulfilled. Then there is an $\varepsilon>0$ such that $w \in \mathcal{A}_{p(\varphi)-\varepsilon}$. The definition of the number $p(\varphi)$ implies that there is a $p_{0}$ such that $p(\varphi)-\varepsilon<p_{0}<p(\varphi)$ and the function $\varphi^{\frac{1}{p_{0}}}$ is quasi-convex. The function $\frac{\varphi(t)}{t^{p_{0}}}$ almost increases by virtue of Lemma 1.1. Therefore for $p_{1}$ with the condition $p(\varphi)-\varepsilon<p_{1}<p$ we have

$$
\begin{gathered}
\int_{0}^{u} \frac{d \varphi(t)}{t^{p_{1}}}=\frac{\varphi(u)}{u^{p_{1}}}+p_{1} \int_{0}^{u} \frac{\varphi(u)}{t^{p_{1}-1}} d t \leq \frac{\varphi(u)}{u^{p_{1}}}+ \\
+p_{1} \frac{\varphi(u)}{u^{p_{0}}} \int_{0}^{u} \frac{d t}{u^{p_{1}-p_{0}-1}}=c \frac{\varphi(u)}{u^{p_{1}}} .
\end{gathered}
$$

On the other hand, since $w \in \mathcal{A}_{p_{1}}$, by Theorem 3.1 we obtain

$$
\begin{gathered}
w\left\{x \in X:\left(\sum_{j=1}^{\infty}\left(\mathbf{M} f_{j}(x)\right)^{\theta}\right)^{1 / \theta}>\lambda\right\} \leq \\
\leq \frac{c}{\lambda^{p_{1}}} \int_{X}\left(\sum_{j}\left|f_{j}(x)\right|^{\theta}\right)^{\frac{p_{1}}{\theta}} w(x) d \mu .
\end{gathered}
$$


At the same time, by the condition of the theorem we have $\varphi \in \Delta_{2}$. Therefore there is a $p$ such that $\frac{\varphi(t)}{t^{p}}$ almost decreases. Setting $p_{2}=\max \{p(\varphi), p\}$, we have

$$
\int_{u}^{\infty} \frac{d \varphi(t)}{t^{p_{2}}} \leq p_{2} \int_{u}^{\infty} \frac{\varphi(t) d t}{t^{p_{2}-1}} \leq c p_{2} \frac{\varphi(u)}{u^{p}} \int_{u}^{\infty} \frac{d t}{t^{p_{2}-p-1}}=\frac{c p_{2}}{p_{2}-p} \frac{\varphi(u)}{u^{p_{2}}} .
$$

Since $p_{2}>p$, the function $w \in \mathcal{A}_{p_{2}}$ and again by Theorem 3.1 we have

$$
\begin{gathered}
w\left\{x \in X:\left(\sum_{j=1}^{\infty}\left(\mathbf{M} f_{j}(x)\right)^{\theta}\right)^{1 / \theta}>\lambda\right\} \leq \\
\leq \frac{c}{\lambda^{p_{2}}} \int_{X}\left(\sum_{j=1}^{\infty}\left|f_{j}(x)\right|^{\theta}\right)^{p_{2} / \theta} w(x) d \mu .
\end{gathered}
$$

For each $\lambda>0$ we write

$$
\begin{aligned}
& { }^{\lambda} f_{j}(x)= \begin{cases}f_{j}(x) & \text { if }\|f(x)\|_{\theta}>\lambda, \\
0 & \text { if }\|f(x)\|_{\theta} \leq \lambda,\end{cases} \\
& \lambda_{j}(x)= \begin{cases}f_{j}(x) & \text { if }\|f(x)\|_{\theta} \leq \lambda, \\
0 & \text { if }\|f(x)\|_{\theta}>\lambda .\end{cases}
\end{aligned}
$$

Assume that ${ }_{\lambda} f=\left({ }_{\lambda} f_{1}, \ldots,{ }_{\lambda} f_{j}, \ldots\right),{ }^{\lambda} f=\left({ }^{\lambda} f_{1}, \ldots,{ }^{\lambda} f_{j}, \ldots\right)$. It is obvious that

$$
\mathbf{M} f_{j}(x) \leq \mathbf{M}_{\lambda} f_{j}(x)+\mathbf{M}^{\lambda} f_{j}(x)
$$

and hence, by Marcinkiewicz' inequality,

$$
\|\mathbf{M} f(x)\|_{\theta} \leq\left\|\mathbf{M}^{\lambda} f(x)\right\|_{\theta}+\left\|\mathbf{M}_{\lambda} f(x)\right\|_{\theta} .
$$

Therefore

$$
\begin{gathered}
\varphi(\lambda) w\left\{x \in X:\|\mathbf{M} f(x)\|_{\theta}>\lambda\right\} \leq \varphi(\lambda) w\left\{x \in X:\left\|\mathbf{M}^{\lambda} f(x)\right\|_{\theta}>\frac{\lambda}{2}\right\}+ \\
+\varphi(\lambda) w\left\{x \in X:\left\|\mathbf{M}_{\lambda} f(x)\right\|_{\theta}>\frac{\lambda}{2}\right\} .
\end{gathered}
$$


Further,

$$
\begin{gathered}
\int_{X} \varphi\left(\|\mathbf{M} f(x)\|_{\theta}\right) w(x) d x \leq \int_{0}^{\infty} w\left\{x \in X:\|\mathbf{M} f(x)\|_{\theta}>\lambda\right\} d \varphi(\lambda) \leq \\
\quad \leq \int_{0}^{\infty} w\left\{x \in X:\left\|\mathbf{M}^{\lambda} f(x)\right\|_{\theta}>\frac{\lambda}{2}\right\} d \varphi(\lambda)+ \\
+\int_{0}^{\infty} w\left\{x \in X:\left\|\mathbf{M}_{\lambda} f(x)\right\|_{\theta}>\frac{\lambda}{2}\right\} d \varphi(\lambda)=I_{1}+I_{2} .
\end{gathered}
$$

Applying (3.9) and (3.8), we obtain

$$
\begin{gathered}
I_{1} \leq c_{1} \int_{0}^{\infty} \frac{2}{\lambda^{p_{1}}}\left(\int_{X}\left\|^{\lambda} f(x)\right\|_{\theta}^{p_{1}} w(x) d x\right) d \varphi(\lambda)= \\
=c_{1} \int_{0}^{\infty} \frac{2}{\lambda^{p_{1}}}\left(\int_{\left\{x:\|f(x)\|_{\theta}>\lambda\right\}}\|f(x)\|_{\theta}^{p_{1}} w(x) d x\right) d \varphi(\lambda)= \\
=c_{1} \int_{X}\|f(x)\|_{\theta}^{p_{1}}\left(\int_{0}^{\|f(x)\|_{\theta}} \frac{d \varphi(\lambda)}{\lambda^{p_{1}}}\right) w(x) d x=c_{1} \int_{X} \varphi\left(\|f(x)\|_{\theta}\right) w(x) d x .
\end{gathered}
$$

Analogously, applying (3.11) and (3.10), we ascertain that the estimate

$$
I_{2} \leq c_{2} \int_{X} \varphi\left(\|f(x)\|_{\theta}\right) w(x) d x
$$

is valid.

\section{$\S$ 4. Weak Type Inequalities for Vector-Valued Maximal FunCTIONS}

This paragraph will be devoted to proving Theorem IV. To this end we need several well-known facts.

Proposition 4.1 (see [19], p. 623). Let $\Omega$ be an open set in $X$. Then there is a sequence $\left(B_{j}\right)=\left(B\left(x_{j}, r_{j}\right)\right)$ such that

(i) $\Omega=\bigcup_{j=1}^{\infty} B_{j}$;

(ii) there exists a constant $\xi \geq 0$ such that

$$
\sum_{j=1}^{\infty} \chi_{B_{j}}(x) \leq \xi ;
$$


(iii) for each $j=1,2, \ldots$, we have $\bar{B}_{j} \cap(X \backslash \Omega) \neq \varnothing$, where $\bar{B}_{j}=$ $B\left(x_{j}, 3 a_{1} r_{j}\right)$ and the constant $\alpha_{1}$ is from the definition of the space $X$.

Proposition 4.2 (see [17], Lemma 1). For each number $a>0$ there is a constant $\alpha_{2}$ such that if $B(x, r) \cap B\left(y, r^{\prime}\right) \neq \varnothing$ and $r \leq \alpha r^{\prime}$, then $B(x, r) \leq B\left(y, a_{2} r^{\prime}\right)$. Note that $a_{2}=a_{1}^{2}(1+a)+a_{0} a_{1} a$.

Proposition 4.3 ([16], Lemma 3.2). If condition (0.8) is fulfilled, then there is a constant $c>0$ such that

$$
\frac{\varphi(s)}{s} \leq c t^{-1} \psi\left(c \frac{t}{\gamma(s)}\right), \quad 0<s \leq t
$$

We start with an extension of Theorem B. The following statement is in fact the sharpening of Theorem 5.1 from [16] for maximal functions in the case $\theta(u) \equiv u, d \beta \equiv w d \mu \otimes \delta_{0}$.

Theorem 4.1. Let $\varphi$ and $\gamma$ be nondecreasing functions defined on $[0, \infty)$, $\psi$ be a quasi-convex function. Further assume that $w, \nu$ and $\sigma$ are weight functions. Then the following statements are equivalent:

(i) there is a positive constant $c_{1}$ such that the inequality

$$
\varphi(\lambda) w\{x: \mathbf{M} f(x)>\lambda\} \leq c_{1} \int_{X} \psi\left(c_{1} \frac{f(x) \nu(x)}{\gamma(\lambda)}\right) \sigma(x) d \mu
$$

is fulfilled for any $\lambda>0$ and locally summable function $f: X \rightarrow R^{1}$;

(ii) there is a positive constant $\varepsilon$ such that

$$
\sup _{B} \sup _{\lambda>0} \frac{1}{\varphi(\lambda) w B} \int_{B} \widetilde{\psi}\left(\varepsilon \frac{\varphi(\lambda) \gamma(\lambda)}{\lambda} \frac{w B}{\mu B \sigma(x) \nu(x)}\right) \sigma(x) d \mu<\infty .
$$

Proof. Since in the proof of Theorem 5.1 the quasi-convexity of $\varphi \gamma$ was used only to show that the implication (i) $\Rightarrow$ (ii) is valid, now it is sufficient to prove this implication by our weakened assumptions.

Let $B$ be a fixed ball and $s>0$. Given $k \in N$, put $B_{k}=\{x \in B$ : $\left.\sigma(x) \nu(x)>\frac{1}{k}\right\}$ and

$$
g(x)=\left(\frac{\varphi(s)}{s} \frac{w B}{\mu B \sigma(x) \nu(x)}\right)^{-1} \widetilde{\psi}\left(\varepsilon \frac{\varphi(s) \gamma(s)}{s} \frac{w B}{\mu B \sigma(x) \nu(x)}\right) \chi_{B_{k}}(x)
$$

with $\varepsilon$ to be specified later. 
In our notation we have

$$
\begin{gathered}
I=\int_{B_{k}} \widetilde{\psi}\left(\varepsilon \frac{\varphi(s) \gamma(s)}{s} \frac{w B}{\mu B \sigma(x) \nu(x)}\right) \sigma(x) d \mu= \\
=\frac{\varphi(s)}{s} \frac{w B}{\mu B} \int_{B} \frac{g(x)}{\nu(x)} d \mu .
\end{gathered}
$$

If $B$ and $s$ are chosen such that

$$
\frac{1}{\mu B} \int_{B} \frac{g(x)}{\nu(x)} d \mu<s
$$

then we obtain the estimate

$$
I \leq \varphi(s) w B
$$

Let now

$$
\frac{1}{\mu B} \int_{B} \frac{g(x)}{\nu(x)} d \mu>s .
$$

By the condition (i) for the function

$$
f(x)=2 s\left(\frac{1}{\mu B} \int_{B} \frac{g(x)}{\nu(x)} d \mu\right)^{-1} \frac{g(x)}{\nu(x)}
$$

and Corollary 1.1 we derive the estimates

$$
\begin{gathered}
I \leq \frac{\varphi(s)}{s} \frac{1}{\mu B} \int_{B} \frac{g(x)}{\nu(x)} w\{x \in X: \mathbf{M} f(x)>s\} d \mu \leq \frac{1}{s} \frac{1}{\mu B} \int_{B} \frac{g(x)}{\nu(x)} d \mu \times \\
\times c_{1} \int_{X} \psi\left(2 c_{1}\left(\frac{1}{\mu B} \int_{B} \frac{g(x)}{\nu(x)} d \mu\right)^{-1} \frac{g(x) s}{\gamma(s)}\right) \sigma(x) d \mu \leq \\
\leq c_{1} \int_{X} \psi\left(2 c_{1} c \frac{g(x)}{\gamma(s)}\right) \sigma(x) d \mu .
\end{gathered}
$$

Therefore

$$
I \leq \varphi(s) w B+c_{1} \int_{X} \psi\left(2 c_{1} c \frac{g(x)}{\gamma(s)}\right) \sigma(x) d \mu .
$$

Choose $\varepsilon$ so small that $2 c_{1} c^{2} \varepsilon<1$. By Corollaries 1.1 and 1.2 and the definition of $g$ we obtain, from the above inequality, the estimate

$$
I \leq \varphi(s) w B+c \varepsilon I
$$


Now we shall show that $I$ is finite for a small $\varepsilon$. Let $\psi(t) \cdot t^{-1} \rightarrow \infty$ as $t \rightarrow \infty$; then $\widetilde{\psi}$ is finite everywhere and thus

$$
I \leq \widetilde{\psi}\left(\varepsilon k \frac{\varphi(s) \gamma(s)}{s} \frac{w B}{\mu B}\right) \sigma B<\infty,
$$

since $\sigma$ and $w$ are locally integrable.

Let now $\psi(t) \leq A t, A>0$. Then the condition (i) implies

$$
\gamma(\lambda) \varphi(\lambda) w\{x \in X: \mathbf{M} f(x)>s\} \leq c \int_{X}|f(x)| \nu(x) \sigma(x) d \mu .
$$

If in this inequality we put $f(x)=s \frac{\mu B}{\mu E} \chi_{E}(x)$, where $E$ is a measurable subset of $B$, we shall obtain the inequality

$$
\frac{\varphi(s) \gamma(s)}{s} \frac{w B}{\mu B} \leq \frac{c}{\mu E} \int_{E} \sigma(x) \nu(x) d \mu
$$

which yields the estimate

$$
\frac{\varphi(s) \gamma(s)}{s} \frac{w B}{\mu B \sigma(x) \gamma(x)} \leq c
$$

almost everywhere on $B$. Here the constant $c$ does not depend on $B$ and $s$. Therefore we conclude that

$$
I \leq \widetilde{\psi}(\varepsilon c) \sigma B
$$

Choosing $\varepsilon$ so small that $\widetilde{\psi}(\varepsilon c)<\infty$, we see that $I$ is finite.

Further, if $c \varepsilon<1$, then inequality (4.2) implies

$$
\int_{B} \widetilde{\psi}\left(\varepsilon \frac{\varphi(s) \gamma(s)}{s} \frac{w B}{\mu B \sigma(x) \nu(x)}\right) \sigma(x) d \mu \leq \frac{1}{1-c \varepsilon} \varphi(s) w B .
$$

Passing here to the limit as $k \rightarrow \infty$, we derive the desired inequality (ii).

In the same manner we can generalize Theorem 5.1 from [16] to its full extent.

Proof of Theorem IV. Let $\lambda>0$ and

$$
\Omega_{\lambda}=\left\{x \in X: \mathbf{M}\left(\|f\|_{\theta}\right)(x)>\lambda\right\} .
$$

Let further $\left(B_{j}\right)_{j}$ be a sequence from Proposition 4.1. We set $G_{\lambda}=X \backslash \Omega_{\lambda}$ and introduce the notation $f_{1}=f \chi_{G_{\lambda}}=\left(f_{1} \chi_{G_{\lambda}}, \ldots, f_{n} \chi_{G_{\lambda}}, \ldots\right), f_{2}=f \chi_{\Omega}$. Condition (0.9) readily implies that $w \in \mathcal{A}_{\infty}$ and therefore $w \in \mathcal{A}_{p}$ for some $p>1$. Let a number $p$ be chosen so that the function $t^{-p} \psi(t)$ almost 
decreases. This is possible due to the condition $\psi \in \Delta_{2}$. As can be easily verified,

$$
\begin{gathered}
\varphi(\lambda) w\left\{x:\|\mathbf{M} f(x)\|_{\theta}>\lambda\right\} \leq \varphi(\lambda) w\left\{x:\left\|\mathbf{M} f_{1}(x)\right\|_{\theta}>\frac{\lambda}{2}\right\}+ \\
+\varphi(\lambda) w\left\{x:\left\|\mathbf{M} f_{2}(x)\right\|_{\theta}>\frac{\lambda}{2}\right\} .
\end{gathered}
$$

By Theorem 3.1

$$
\varphi(\lambda) w\left\{x:\left\|\mathbf{M} f_{1}(x)\right\|_{\theta}>\frac{\lambda}{2}\right\} \leq \frac{c \varphi(\lambda)}{\lambda^{p}} \int_{G_{\lambda}}\|f(x)\|_{\theta}^{p} w(x) d \mu .
$$

Next, since $\|f(x)\|_{\theta} \leq \lambda$ for $x \in G_{\lambda}$, from (4.1) and the $\Delta_{2}$-condition we obtain the estimate

$$
\begin{aligned}
& \frac{\varphi(\lambda)}{\lambda^{p}} \int_{G_{\lambda}}\|f(x)\|_{\theta}^{p} w(x) d \mu \leq c \int_{g_{\lambda}} \frac{\psi\left(c \frac{\lambda}{\gamma(\lambda)}\right)}{\lambda^{p}}\|f(x)\|_{\theta}^{p} w(x) d \mu \leq \\
& \leq c \int_{G_{\lambda}} \psi\left(c \frac{\|f(x)\|_{\theta}}{\gamma(\lambda)}\right) w(x) d \mu \leq c \int_{X} \psi\left(\frac{\|f(x)\|_{\theta}}{\gamma(\lambda)}\right) w(x) d \mu .
\end{aligned}
$$

Therefore (4.4) implies

$$
\varphi(\lambda) w\left\{x \in X:\left\|\mathbf{M} f_{1}(x)\right\|_{\theta}>\frac{\lambda}{2}\right\} \leq c \int_{X} \psi\left(\frac{\|f(x)\|_{\theta}}{\gamma(\lambda)}\right) w(x) d \mu .
$$

We set $\tilde{f}=\left(\widetilde{f}_{1}, \ldots, \widetilde{f}_{j}, \ldots\right)$, where

$$
\widetilde{f}_{j}(x)=\sum_{k}\left(\frac{1}{\mu \bar{B}_{k}} \int_{\bar{B}_{k}}\left|f_{j}(y)\right| d \mu\right) \chi_{B_{k}}(x) .
$$

Let $\widetilde{B}_{k}=B\left(x_{k}, 2 a_{1} r_{k}\right)$. We set $\widetilde{\Omega}_{\lambda}=\cup_{k} \widetilde{B}_{k}$ and $\widetilde{G}_{\lambda}=X \backslash \widetilde{\Omega}_{\lambda}$.

Now it will be shown that

$$
\mathbf{M}\left(f_{j} \chi_{\Omega_{\lambda}}\right)(x) \leq c \mathbf{M} \widetilde{f}_{j}(x) \quad(j=1,2, \ldots)
$$

for $x \in \widetilde{G}_{\lambda}$.

Let $x \in \widetilde{G}_{\lambda}$ and $B=B(y, r)$ be an arbitrary ball containing the point $x$ and $B \cap \Omega_{\lambda} \neq \varnothing$. It will be shown that for an arbitrary $k \in S, S=\{k \in$ $\left.\mathbb{N}: B_{k} \cap B \neq \varnothing\right\}$, we have $B_{k} \subset a_{2} B$, where $a_{2}$ is an absolute constant not depending on $k$. Since $x \in \widetilde{G}_{\lambda}$, it is obvious that $x \in B \backslash \widetilde{B}_{k}$. Therefore

$$
d\left(x_{k}, x\right)>2 a_{1} r_{k} .
$$


Let $z \in B_{k} \cap B$. We have

$$
d(z, x) \leq a_{1}(d(z, y)+d(y, x)) \leq a_{1}\left(a_{0}+1\right) r
$$

and

$$
2 a_{1} r_{k} \leq d\left(x_{k}, x\right) \leq a_{1}\left(d\left(x_{k}, z\right)+d(z, x)\right) \leq a_{1}\left(r_{k}+a_{1}\left(a_{0}+1\right) r\right) .
$$

Hence it follows that $r_{k} \leq a_{1}\left(a_{0}+1\right) r$. Now on account of Proposition 4.2 we have $B_{k} \subseteq a_{2} B$, where $a_{2}=a_{1}^{2}\left(a_{1}\left(a_{0}+1\right)\right)+a_{0} a_{1}^{2}\left(a_{0}+1\right), a_{2} B=$ $B\left(y, a_{2} r\right)$.

By virtue of the latter inclusion and doubling condition for $\mu$ we derive the inequalities

$$
\begin{aligned}
& \frac{1}{\mu B} \int_{B} f_{j} \chi_{\Omega_{\lambda}}(x) d \mu=\frac{1}{\mu B} \sum_{k \in S} \int_{B \cap B_{k}} f_{j}(y) d \mu \leq \\
& \leq \frac{1}{\mu B} \sum_{k \in S} \int_{\bar{B}_{k}} f_{j}(y) d \mu \leq \frac{c}{\mu a_{2} B} \sum_{k \in S}\left(\frac{1}{\mu \bar{B}_{k}} \int f_{\bar{B}_{k}} f_{j}(y) d \mu\right) \mu \bar{B}_{k} \leq \\
& \leq \frac{c}{\mu a_{2} B} \int_{a_{2} B}\left(\sum_{k}\left(\frac{1}{\mu \bar{B}_{k}} \int_{\bar{B}_{k}} f_{j}(y) d \mu\right)\right) \chi_{B_{k}} d \mu \leq \\
& \leq \frac{c}{\mu a_{2} B} \int_{a_{2} B} \widetilde{f}_{j}(y) d \mu \leq \mathbf{M} \widetilde{f}_{j}(x),
\end{aligned}
$$

thereby proving (4.6).

Taking (4.3) into account, we obtain

$$
\begin{gathered}
\varphi(\lambda) w\left\{x \in X:\left\|\mathbf{M} f_{2}(x)\right\|_{\theta}>\frac{\lambda}{2}\right\} \leq \varphi(\lambda) w \widetilde{\Omega} \widetilde{\Omega}_{\lambda}+ \\
+\varphi(\lambda) w\left\{x \in \widetilde{G}_{\lambda}:\|\mathbf{M} \widetilde{f}(x)\|_{\theta}>c \lambda\right\} .
\end{gathered}
$$

Since condition (0.8) ensures the belonging of the function $w$ to the class $\mathcal{A}_{\infty}$, this function will satisfy the doubling condition. Therefore

$$
w \widetilde{\Omega}_{\lambda} \leq \sum_{k=1}^{\infty} w \widetilde{B}_{k} \leq c_{1} \sum_{k=1}^{\infty} w B_{k} \leq c_{1} \int_{\substack{\cup B_{k} \\ k}} \sum_{k} \chi_{B_{k}} d \mu \leq c_{1} \xi w \Omega_{\lambda} .
$$

Further by virtue of Theorem 3.1 we have

$$
\begin{gathered}
\varphi(\lambda) w\left\{x \in \widetilde{G}_{\lambda}:\|\mathbf{M} \tilde{f}(x)\|_{\theta}>c \lambda\right\} \leq \\
\leq c_{2} \frac{\varphi(\lambda)}{\lambda^{p}} \int_{\Omega_{\lambda}}\|\widetilde{f}(x)\|_{\theta}^{p} w(x) d \mu .
\end{gathered}
$$


Applying the Minkowski inequality and taking into account that $\bar{B}_{k} \cap G_{\lambda} \neq$ $\varnothing$ and $\mathbf{M}\left(\|f(x)\|_{\theta}\right)(z) \leq \lambda$ for $z \in G_{\lambda}$, we find that for $x \in \Omega_{\lambda}$

$$
\begin{aligned}
& \|\widetilde{f}(x)\|_{\theta}=\left(\sum_{j=1}^{\infty}\left|\tilde{f}_{j}(x)\right|^{\theta}\right)^{1 / \theta}= \\
& =\left(\sum_{j=1}^{\infty}\left(\sum_{k} \frac{1}{\mu \bar{B}_{k}} \int_{\bar{B}_{k}}\left|f_{j}(y)\right| d \mu \chi_{B_{k}}(x)\right)^{\theta}\right)^{1 / \theta} \leq \\
& =\sum_{k=1}^{\infty}\left(\sum_{j=1}^{\infty}\left(\frac{1}{\mu \bar{B}_{k}} \int_{\bar{B}_{k}}\left|f_{j}(y)\right| d \mu \chi_{B_{k}}(x)\right)^{\theta}\right)^{1 / \theta} \leq \\
& =\sum_{k=1}^{\infty} \frac{1}{\mu \bar{B}_{k}}\left(\sum_{j=1}^{\infty}\left(\int_{\bar{B}_{k}}\left|f_{j}(y)\right| d \mu\right)^{\theta}\right)^{1 / \theta} \chi_{B_{k}}(x) \leq \\
& =\sum_{k=1}^{\infty} \frac{1}{\mu \bar{B}_{k}}\left(\int_{\bar{B}_{k}}\left(\sum_{j=1}^{\infty}\left|f_{j}(y)\right|^{\theta}\right)^{1 / \theta} d \mu\right) \chi_{B_{k}}(x)= \\
& =\sum_{k=1}^{\infty}\left(\frac{1}{\mu \bar{B}_{k}} \int_{\bar{B}_{k}}\|f(x)\|_{\theta} d \mu\right) \chi_{B_{k}}(x) \leq \lambda \sum_{k=1}^{\infty} \chi_{B_{k}}(x) \leq \xi \lambda .
\end{aligned}
$$

Thus (4.9) implies

$$
\varphi(\lambda) w\left\{x \in \widetilde{G}_{\lambda}:\|\mathbf{M} \widetilde{f}(x)\|_{\theta}>c \lambda\right\} \leq c_{3} \varphi(\lambda) w \Omega_{\lambda} .
$$

Due to the latter estimate (4.7) yields

$$
\varphi(\lambda) w\left\{x \in X:\left\|\mathbf{M} f_{2}(x)\right\|_{\theta}>\frac{\lambda}{2}\right\} \leq c_{3} \varphi(\lambda) w \Omega_{\lambda} .
$$

By virtue of the respective result in the scalar case (see Theorem 4.1) we have

$$
\varphi(\lambda) w \Omega_{\lambda} \leq c_{4} \int_{X} \psi\left(\frac{\|f(x)\|_{\theta}}{\gamma(\lambda)}\right) w(x) d \mu .
$$

Now, from (4.3), (4.5), (4.10), (4.11) we obtain the validity of the desired inequality. 


\section{REFERENCES}

1. D. Gallardo, Orlicz spaces for which the Hardy-Littlewood maximal operator is bounded. Publ. Mat. 32(1938), 261-266.

2. A. Gogatishvili, V. Kokilashvili and M. Krbec, Maximal functions in the classes $\varphi(L)$. (Russian) Dokl. AN SSSR 314(1990), No.3, 534-536; English translation: Soviet Math. Dokl. 42(1991), No.2.

3. V. Kokilashvili and M. Krbec, Weighted inequalities in Lorentz and Orlicz spaces. World Scientific. Singapore, New York, London, Hong Kong. 1991.

4. A. Gogatishvili, V. Kokilashvili and M. Krbec, Maximal functions, $\varphi(L)$ classes and Carleson measures. Proc. Razmadze Math. Inst. Georgian Acad. Sci. 102(1993), 85-97.

5. R. Kerman and A. Torchinsky, Integral inequalities with weights for the Hardy maximal functions. Studia Math. 31(1982), 247-284.

6. L. Pick, Weighted estimates for the Hilbert transforms of odd functions. Proc. Georgian Acad. Sci. Math. 1(1993), No. 1, 87-107.

7. S. Bloom and R. Kerman, Weighted Orlicz space integral inequalities for the Hardy-Littlewood maximal operator. Preprint.

8. V. Kokilashvili, On traces of functions with partial derivatives from Orlicz classes. Comment. Math. Special issue (Tomus specialis in honorem Ladislai Orlicz). PWN, Polish Acad. Sci., Warsaw, 1978, 183-189.

9. C. Fefferman and E.M. Stein, Some maximal inequalities. Amer. J. Math. 93(1971), 107-115.

10. V.M. Kokilashvili, Maximal inequalities and multipliers in weighted Lizorkin-Triebel spaces. (Russian) Dokl. Akad. Nauk SSSR, 239(1978), 42 -45; English translation:Soviet Math. Dokl. 19(1978), 272-276.

11. "—_, Maximal functions in weighted spaces. (Russian) Trudy Tbiliss. Mat. Inst. Razmadze 65(1980), 110-121.

12. "__, Maximal functions and singular integrals in weighted functions spaces. (Russian) Metsniereba, Tbilisi, 1985.

13. H.P. Heinig and R. Johnson, Weighted norm inequalities for $L^{r}$ valued integral operators and applications. Math. Nachr. 107(1982), 161174 .

14. L. Pick, Two weight weak type inequalities in Orlicz classes. Studia Math. 100(1991) No. 3, 207-218.

15. A. Gogatishvili and L. Pick, Weighted inequalities for weak and extraweak type for the maximal operator and Hilbert transform, Czechoslovak Math. J. 43(118)(1993), No.3, 547-566.

16. A. Gogatishvili, General weak type inequalities for the maximal operators and singular integrals. Proc. Razmadze Math. Inst. (Trudy Tbiliss. Mat. Inst. Razmadze) 101(1992), 47-63. 
17. J.O. Strömberg and A. Torchinsky, Weighted Hardy spaces. Lecture Notes in Math. 1381, Springer-Verlag, New-York, 1989.

18. J.O. Strömberg, Bounded mean oscillation with Orlicz norms and duality of Hardy spaces. Indiana Univ. Math. J. 28(1979), 511-544.

19. R. Coifman and G. Weiss, Extensions of Hardy spaces and their use in Analysis. Bull. Amer. Math. Soc. 83(1977) No. 4, 569-644.

20. R.A. Macías and C. Segovia, A well quasi-distance for spaces of homogeneous type. Trab. Mat. Inst. Argentina Mat. 32(1981), 1-18.

21. J. Genebashvili, Weighted estimates in Lorentz spaces for a maximal function given on spaces of the homogeneous type. Proc. Razmadze Inst. 106(1993), 49-61.

22. J. Genebashvili, A. Gogatishvili and V. Kokilashvili, Criteria of general weak type inequalities for integral transforms with positive kernels. Proc. Georgian Acad. Sci. Math. 1(1993), No. 1, 11-34.

23. J. Garcia-Cuerva and J.L. Rubio de Francia, Weighted norm inequalities and related topics. North Holland, Amsterdam, 1985.

24. A. Benedec and R. Panzone, The spaces $L^{p}$ with mixed norm. Duke Math. J. 28(1961), No. 3, 301-324.

25. H.Aimar and R.A. Macías, Weighted norm inequalities for the HardyLittlewood maximal operator on spaces of homogeneous type. Proc. Amer. Math. Soc. 91(1984), No. 2, 213-216.

(Received 6.09.1993)

Authors' address:

A. Razmadze Mathematical Institute Georgian Academy of Sciences

1, Z. Rukhadze St., Tbilisi, 380093

Republic of Georgia 